\title{
DETECÇÃO DE FALHAS EM ESTRUTURAS INTELIGENTES USANDO OTIMIZAÇÃO POR NUVEM DE PARTÍCULAS: FUNDAMENTOS E ESTUDO DE CASOS
}

\author{
Adriano Tebaldi* \\ atebaldidtecumseh.com.br
}

Leandro dos Santos Coelho ${ }^{\dagger}$

leandro.coelho@pucpr.br

\author{
Vicente Lopes Junior* \\ vicente@dem.feis.unesp.br
}

*Universidade Estadual Paulista - UNESP - Departamento de Engenharia Mecânica

Av. Brasil Centro, 56, CEP 15.385.000, Ilha Solteira, SP, Brasil

${ }^{\dagger}$ Grupo Produtrônica - Programa de Pós-Graduação em Engenharia de Produção e Sistemas

PUCPR / CCET / PPGEPS / LAS - Laboratório de Automação e Sistemas, Centro de Ciências Exatas e de Tecnologia

Pontifícia Universidade Católica do Paraná, Rua Imaculada Conceição, 1155, CEP 80215-901, Curitiba, PR, Brasil

\section{RESUMO}

Os materiais piezelétricos são denominados de "materiais inteligentes" e pertencem a uma classe de dielétricos que exibem deformação significativa em resposta a aplicação de um campo elétrico. Estes materiais, também, produzem uma polarização dielétrica, ou seja, um campo elétrico em resposta a deformação no material. Esta dupla propriedade exibida pelos materiais piezelétricos torna extremamente vantajosa a aplicação destes materiais para sistemas de controle e para localização e caracterização de falhas estruturais. Neste artigo, a caracterização de falhas estruturais é realizada em dois passos. Em uma primeira etapa é utilizado o método da impedância elétrica para se determinar a região do dano e em uma segunda etapa se utiliza um método de otimização para quantificar a severidade das falhas. Identificação de falhas pertence ao grupo de problemas inversos, e portanto, não há solução única. A metodologia híbrida proposta se beneficia da técnica de impedância elétrica para localizar as regiões de danos e, assim, diminuir o número de variáveis envolvidas

\footnotetext{
Artigo submetido em 01/08/2005

1a. Revisão em 24/05/2006

2a. Revisão em 05/09/2006

Aceito sob recomendação do Editor Associado

Prof. Ivan Nunes Da Silva
}

no processo de otimização. O procedimento é validado através de diferentes abordagens de otimização por nuvem de partículas, que é uma técnica da inteligência coletiva, usando operadores com geração de números aleatórios baseados em distribuições Gaussiana e de Cauchy.

PALAVRAS-CHAVE: sensor e atuador piezelétrico, impedância elétrica, inteligência coletiva, nuvem de partículas, otimização de sistemas, detecção de falhas.

\section{ABSTRACT}

Piezoelectric materials exhibit significant deformation in response to an applied electric field, as well as generating an electrical charge in response to mechanical strain. Control designs and characterization of structural damage can take advantage of these dual properties. In this paper, the identification of damage is realized in two steps. The first uses the electric impedance technique in order to determine the location of the damage, and the second uses an optimization method to quantify the severity of the damage. Damage identification is an inverse problem, and has no unique solution. The hybrid approach proposed in this paper takes advantage of the electric impedance technique to localize the damaged 
regions with accuracy. This information permits a decrease in the number of variables involved in the process, which is a goal for any optimization technique. The procedure is validated through different particle swarm optimization approaches using an operator with random numbers based on Gaussian and Cauchy distributions.

KEYWORDS: piezoelectric sensor and actuator, smart structure, electric impedance, swarm intelligence, particle swarm optimization, system optimization, fault detection.

\section{INTRODUÇÃO}

A manutenção preditiva de máquinas e equipamentos vem encontrando aceitação crescente na indústria. Manutenção preditiva consiste basicamente em monitorar parâmetros que caracterizam a condição de estruturas ou máquinas de forma a poder detectar, prever a época provável de ocorrência, e se possível, diagnosticar o tipo de falha para que se possa planejar a operação de manutenção na ocasião e de forma conveniente (Tebaldi, 2004).

As falhas ocorridas em equipamentos e estruturas podem ser devidas a atrito, fadiga, impacto, crescimento de trinca ou alguma outra razão. Para um adequado funcionamento do sistema, a falha deve ser localizada e reparada, se possível. O problema de detecção de falhas está compreendido em localizar, quantificar e estimar a vida útil restante. O local onde a falha está situada pode ter um efeito catastrófico na estrutura. Um monitoramento periódico das condições da estrutura é necessário para avaliar a integridade estrutural. Devido aos avanços nos equipamentos de processamento de sinal, a técnica de detecção de falhas tem evoluído significativamente nas últimas décadas. Os métodos de detecção de falhas podem ser classificados em dois grupos (Kabeya, 1998): métodos baseados em modelos matemáticos ou quantitativos e métodos qualitativos (não necessitam de modelo) (Chen e Patton, 1993).

O diagnóstico de falhas é um processo de avaliação de danos que pode ser pensado de duas maneiras: (i) monitoramento das mudanças de condição e, se necessário, parar a operação do equipamento antes de piorar as condições de operação; e (ii) garantir que o dano iniciado não se estenda para uma situação de risco. As técnicas de obtenção de (i) estão melhores definidas que as de (ii).

Uma importante técnica de monitoramento de estruturas é a técnica da impedância elétrica. A concepção básica desta técnica é o uso de altas freqüências de vibrações, as quais excitam modos locais da estrutura. Isto permite a identificação de falhas incipientes. Esta metodologia é baseada na utilização de sensores/atuadores piezelétricos, os quais fornecem medidas diretamente relacionadas com a impedância mecâ- nica da estrutura. As medidas de impedância elétrica são, geralmente, realizadas em altas freqüências, maiores que 20 $\mathrm{kHz}$. O pequeno comprimento de onda nessas freqüências permite a detecção de pequenas mudanças na integridade estrutural. A técnica de monitoramento da condição da estrutura baseada na impedância elétrica é um método promissor de avaliação não-destrutiva.

O monitoramento da saúde estrutural (structural health monitoring) tem sido definido na literatura como aquisição, validação e análise de dados técnicos para facilitar as decisões no gerenciamento do número de ciclos de vida útil. O desafio que vem agregando pesquisadores é fornecer informações quantitativas dos danos presentes na estrutura. Para isto técnicas de otimização computacional (Doebling et al., 1998), redes neurais artificiais (Kaminski, 1997; Lopes Jr. et al., 2000; Patton et al., 2000) e algoritmos evolutivos (Friswell et al., 1998; He et al., 2001; Simões e Steffen, 2002; Tebaldi et al., 2003; Tebaldi et al., 2005) têm sido utilizados.

Neste contexto, alguns trabalhos recentes devem ser comentados. Xu et al. (2004) e Yang et al. (2005) descreveram uma técnica de monitoramento da saúde estrutural usando uma abordagem de otimização de programação evolutiva híbrida com método de avaliação não-destrutiva baseado em impedância. Os resultados apresentados demonstraram que o método proposto é eficiente em localizar e quantificar falhas em uma barra e também em uma placa. Tseng e Wang (2005) apresentaram uma técnica de identificação de danos estruturais baseada no método da impedância e usando sensores piezoelétricos. Os resultados foram promissores para um estudo de caso considerando uma barra com sensores. Marwala e Chakraverty (2006) utilizaram redes neurais autoassociativas combinadas com algoritmos genéticos para classificação de falhas em diversas estruturas cilíndricas. Os resultados de classificação apresentaram um desempenho entre 91 e $96 \%$ de classificações corretas.

O prognóstico de danos, que é a previsão em tempo real da vida útil restante do sistema, é um desafio atual que poderá alterar a concepção desta área. Este assunto é abordado com detalhes em Inman et al. (2005).

Dentre as técnicas emergentes da inteligência computacional que podem ser utilizadas para quantificar as falhas estruturais, pode-se citar as abordagens da inteligência coletiva. A inteligência coletiva (swarm intelligence) apresenta características bio-inspiradas de população e evolução similares aos algoritmos evolutivos (ou evolucionários), contudo, diferencia-se por enfatizar o comportamento de cooperação entre grupos de membros. A inteligência coletiva é utilizada para resolver problemas de otimização e cooperação de agentes inteligentes em problemas de otimização, tais como em eletromagnetismo (Robinson e Samii, 2004), planejamento 
de sistemas elétricos (Kannan et al., 2004), otimização de forma (Fourie e Groenwold, 2002), robótica coletiva (Liu e Passino, 2004) e controle cooperativo e/ou descentralizado (Baras et al., 2003). A inteligência coletiva é inspirada na natureza, no fato de que a interação entre os diversos membros de um determinado grupo de seres vivos contribui com as suas próprias experiências para o grupo, tornando-o mais forte perante os outros. Os representantes mais conhecidos da inteligência coletiva em problemas de otimização são abordagens de colônia de formigas (Dorigo e Di Caro, 1999), nuvem ou bando de partículas (Kennedy e Eberhart, 2001), colônia de bactérias (Müller et al., 2002) e sistema imunológico artificial (de Castro, 2001).

Neste trabalho são abordadas concepções de projeto de nuvem de partículas (particle swarm optimization, PSO) para quantificar as falhas em estruturas inteligentes. Abordagens de PSO para identificação de falhas podem ser utilizadas com maior sucesso se combinadas com a técnica da impedância elétrica. Esta abordagem híbrida é desenvolvida neste trabalho. A técnica de impedância elétrica é adotada para detectar com exatidão a região da falha, pois utiliza altas faixas de frequencia, que excitam os modos locais de vibração da estrutura. Se a região da falha é conhecida, o número de parâmetros a serem adotados no processo de otimização pode ser reduzido, assim o algoritmo terá maior chance de convergir para a solução ótima, ou seja, detectar a severidade da falha. Em síntese, este trabalho focaliza a caracterização das falhas em estruturas englobando duas etapas. Na primeira etapa é utilizado o método da impedância elétrica para detectar se existe falha e, em caso positivo qual a região que ela se encontra. Na segunda etapa se utiliza nuvem de partículas para quantificar a severidade falha.

O artigo é organizado da seguinte forma. Na seção 2, alguns conceitos e características vinculados a impedância elétrica são apresentados e discutidos. As abordagens de PSO utilizadas para quantificar a falha são descritas em detalhes na seção 3. Na seção 4 é abordada a metodologia de análise. A análise dos resultados obtidos e as conclusões são apresentadas nas seções 5 e 6 , respectivamente.

\section{DETECÇÃO DE FALHAS}

As estruturas ou sistemas mecânicos, às vezes, são submetidos a grandes esforços, os quais podem levar a uma deterioração das condições originais de trabalho. Estes danos podem ser devidos a fadiga, impacto, evolução de trincas ou algum outro motivo e causam incômodos como, por exemplo, o aumento das vibrações. A necessidade de monitorar e reparar estruturas tem originado diferentes técnicas de detecção de falhas. As primeiras investigações em detecção de falhas, a partir de dados de vibrações, foram publicadas bem antes do estabelecimento de análise modal experimental como uma técnica popular e prática. Uma revisão geral dos métodos de detecção da falhas baseados em dados de vibrações foi apresentada por Friswell e Penny (1997). Foram abordados métodos que utilizam três tipos básicos de dados para medidas de vibrações dinâmicas: domínio do tempo, domínio da frequiência e domínio modal. Doebling et al. (1998) realizaram uma revisão dos métodos de detecção, localização e caracterização de falhas em estruturas e sistemas mecânicos a partir da análise de medidas da resposta de vibração.

O problema de detecção de falhas é classificado como um problema inverso e consiste em determinar as causas tendo como referência os efeitos. Em problemas inversos os parâmetros de falha (comprimento e/ou localização) podem ser calculados através da variação da freqüência natural. Entretanto, os problemas diretos consistem na determinação da solução a partir da completa descrição das causas (Tebaldi, 2004).

Neste contexto, na subseção a seguir são apresentados alguns conceitos de materiais piezelétricos e a técnica de detecção de falhas baseada em impedância elétrica.

\subsection{Detecção de falhas baseada na téc- nica de impedância elétrica}

Embora existam diversas definições para o termo inteligente (smart), a comunidade de engenharia tem adotado o termo de estruturas inteligentes (smart structures) desde o início da década 90 para descrever estruturas ou componentes estruturais com habilidades especiais e, que não são encontradas em materiais/estruturas convencionais.

A ciência de estruturas inteligentes é recente e nesta conjuntura podemos ter uma visão desta tecnologia como uma oportunidade para resultados em longo prazo, ou podemos examinar os impactos que as estruturas inteligentes terão na habilidade do sistema para exibir adaptação em curto prazo. O estudo da tecnologia de estruturas inteligentes é importante devido ao potencial demonstrado para estruturas convencionais em várias aplicações de controle ativo (Clark et al., 1998), controle adaptativo (Silcox et al., 1992) e automonitoramento de condições ou estruturas (Lopes $\mathrm{Jr}$ et al., 2000).

Usualmente, emprega-se o termo de sistemas de materiais inteligentes a aqueles compostos de materiais piezelétricos, eletro-resistivo e magneto-resistivo, fluidos e sólidos eletroreológico, ligas de memória de forma, ou fibras óticas. As propriedades de alguns destes materiais são conhecidas há longo tempo, como por exemplo, o efeito piezelétrico que foi descrito pela primeira vez em 1880 por Pierre e Jacques Curie. 


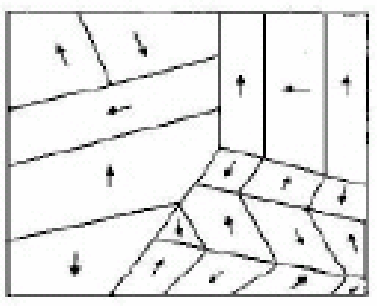

antes da polarização

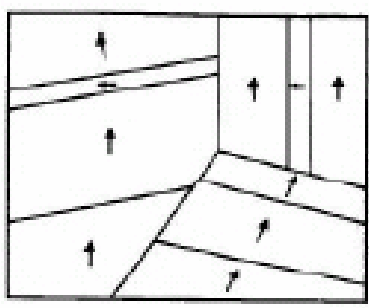

depois da polarização (a)
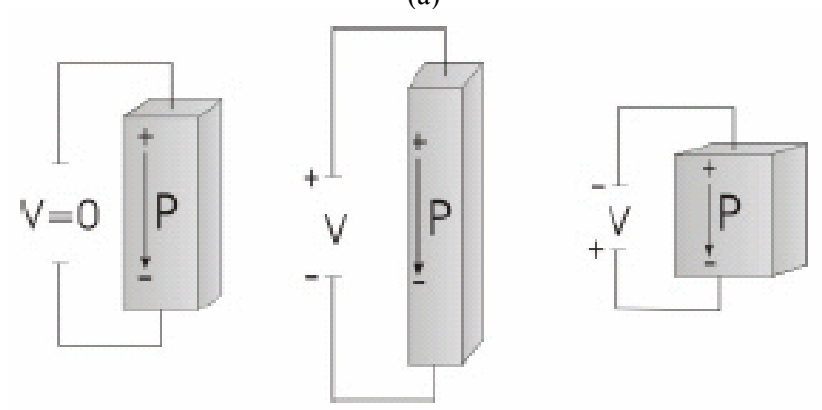

(b)

gimento de deformações mecânicas que, por sua vez, ocasionam variações nas características estáticas e dinâmicas (rigidez e freqüências naturais de vibração, por exemplo). No segundo caso, os elementos piezelétricos, geralmente dispostos sob a forma de placas ou películas finas, podem ser colados na superfície da estrutura, ou embutidos, e recebem destas as deformações mecânicas geradas por vibrações e/ou carregamentos externos. Estas deformações provocam o surgimento de cargas elétricas no material piezelétrico, os quais constituem o sinal de saída do sensor.

A característica que torna extremamente vantajoso o uso dos materiais piezelétricos é a possibilidade de construção de sensores e atuadores distribuídos ao longo de superfícies relativamente amplas, o que tem importantes implicações no tocante a sensibilidade dos sensores, a capacidade de aplicação de cargas dos atuadores e a potência requerida na atuação.

Diversos tipos de materiais, naturais e sintéticos, exibem propriedades piezelétricas. Dentre eles, pode-se citar:

- Cerâmicas piezelétricas. Em particular, as cerâmicas PZT (Titanato Zirconato de Chumbo), têm sido extensivamente utilizadas como elementos atuadores distribuídos, aplicados sob a forma de placas, com espessuras típicas da ordem de 0,1 a 0,2 mm;

- Polímeros piezelétricos. O PVDF (Fluorido de Polivinilideno) tem tido larga utilização como sensores distribuídos, aplicados sob a forma de filmes finos, com espessura típica da ordem de 40 a $120 \mu \mathrm{m}$.

Os materiais piezelétricos, abordados neste trabalho, são os materiais mais utilizados em sistemas adaptativos (Banks et al., 1996). Eles possuem a propriedade de sofrer polarização (surgimento de cargas elétricas) quando são deformados por esforços mecânicos externos (efeito piezelétrico direto). Inversamente estes materiais têm sua forma alterada quando seus dipolos se alinham espontaneamente sob ação de um campo elétrico externo, causando deformação da estrutura cristalina (efeito piezelétrico inverso). Em outras palavras: quando uma tensão elétrica é aplicada na mesma direção da polarização, o material alonga na direção polar e ocorre uma contração transversal. Quando a tensão elétrica é aplicada oposta à direção de polarização, o material contrai na direção polar e expande na direção transversal. Este efeito pode ser observado na figura 1.

O efeito piezelétrico inverso é explorado na construção de atuadores, ao passo que o efeito direto é utilizado na concepção de sensores. No primeiro caso, os elementos piezelétricos, geralmente dispostos sob forma de placas finas, são colados rigidamente na estrutura elástica. A aplicação de tensão elétrica ao material piezelétrico gera deformações que são transferidas para a estrutura, provocando nesta o sur-

Esta seção descreve a técnica em que o atuador e sensor são combinados num simples elemento piezelétrico chamado auto-sensor-atuador. O auto-sensor-atuador tem um número de propriedades desejáveis, não facilmente obtidos com sensores e atuadores separados (Inman, 1990). O atuador é integrado na estrutura e a tensão, deformação, campo elétrico e deslocamento elétrico, dentro do material piezelétrico podem ser completamente descritos por um par de equações eletromecânicas.

As propriedades piezelétricas são definidas utilizando-se três eixos de direções, sendo duas destas direções isotrópicas (mesmas propriedades em todos os pontos destas direções) e a outra é associada com a direção de polarização, que sofre o efeito piezelétrico, conforme apresentado na figura 2. Os sensores e atuadores piezelétricos podem ser criados pela polarização de material apropriado através da aplicação de um campo elétrico a altas temperaturas. A polarização tem efeito de alinhar parcialmente os eixos polares para se obter um alinhamento macroscópico que resulte no acoplamento eletro-mecânico. Como resultado deste acoplamento, o ma- 


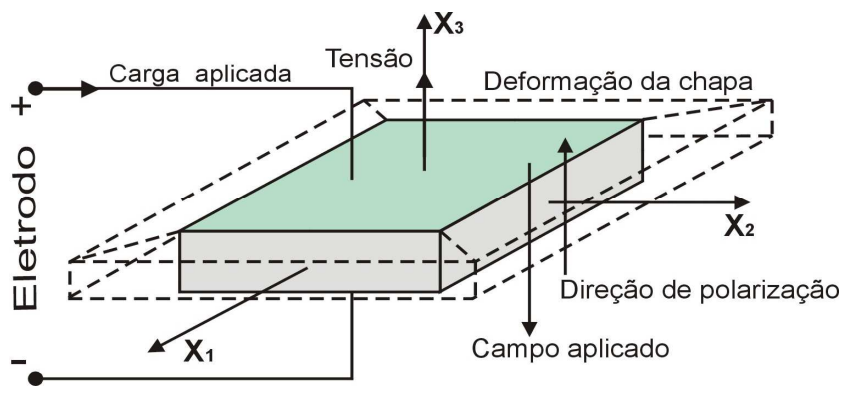

Figura 2: Direções dos eixos para materiais piezelétricos.

terial piezelétrico se deforma em resposta a aplicação de um campo elétrico, dando ao material a propriedade de atuador. A capacidade de sensor do material é obtida através do efeito inverso, no qual as tensões mecânicas no material causam uma rotação parcialmente alinhada nos dipolos para gerar um campo elétrico.

Os elementos piezelétricos apresentam histerese não linear para elevados níveis de excitação, no entanto, para aplicações estruturais comuns podem ser aproximadas para lineares. Existem vários pares de equações eletromecânicas e a escolha depende do problema. Usando a notação padrão IEEE 176-1987, a tensão, a deformação e o deslocamento elétrico de um material piezelétrico podem ser descritos pelas equações (1) e (2). As letras nos colchetes indicam as unidades das variáveis no Sistema Internacional de Unidades, com Newton (N), metro (m), tensão (V) e Coulomb (C), (Clark et al., 1998).

$$
\begin{aligned}
& \{D\}=[e]^{T}\{S\}+\left[\varepsilon^{S}\right]\{E\} \quad \text { (efeito direto) } \\
& \{T\}=\left[C^{E}\right]\{S\}-[e]\{E\} \quad \text { (efeito inverso) }
\end{aligned}
$$

onde o expoente ( $)^{S}$ significa que os valores são medidos para deformação constante e o expoente ( $)^{E}$ significa que os valores são medidos para um campo elétrico constante, $\{T\}$ é o tensor de tensão $\left[\mathrm{N} / \mathrm{m}^{2}\right],\{D\}$ é o vetor de deslocamento elétrico $\left[\mathrm{C} / \mathrm{m}^{2}\right],\{S\}$ é o tensor deformação $[\mathrm{m} / \mathrm{m}],\{E\}$ é o campo elétrico $[\mathrm{V} / \mathrm{m}=\mathrm{N} / \mathrm{C}],\left[C^{E}\right]$ é o tensor de elasticidade para campo elétrico constante $\left[\mathrm{N} / \mathrm{m}^{2}\right]$, [e] é o tensor de permissividade dielétrica $\left[\mathrm{N} \cdot \mathrm{m} / \mathrm{V} \cdot \mathrm{m}^{2}=\mathrm{C} / \mathrm{m}^{2}\right]$ e $\left[\varepsilon^{S}\right]$ é o tensor dielétrico para deformação mecânica constante $\left[\mathrm{N} . \mathrm{m} / \mathrm{V}^{2} \cdot \mathrm{m}\right]$.

Se cada elemento da matriz constante de material piezelétrico, [ $e$ ], é designado por $e_{i j}$ onde $i$ corresponde às linhas, e $j$ às colunas da matriz, então $e_{i j}$ corresponde à tensão desenvolvida na $j$-ésima direção devido a um campo elétrico aplicado na $i$-ésima direção. As constantes piezelétricas de tensão podem ser obtidas a partir das constantes da deformação, usando as equações constitutivas lineares opcionais:

$$
\begin{gathered}
\{S\}=\left[s^{E}\right]\{T\}+[d]\{E\} \\
\{D\}=[d]^{T}\{T\}+\left[\varepsilon^{T}\right]\{E\}
\end{gathered}
$$

onde $\left[\varepsilon^{T}\right]$ é o tensor dielétrico para tensão constante e $[d]$ é uma matriz de constantes piezelétrica. A equação (3) é denominada de equação do atuador e a equação (4), de equação do sensor. A equação de movimento para um PZT vibrando na direção $y$, pode ser expressa como:

$$
\rho \frac{\partial^{2} v}{\partial t^{2}}=\bar{Y}_{22}^{E} \frac{\partial^{2} v}{\partial y^{2}}
$$

onde $\nu$ é o deslocamento na direção $y, \bar{Y}_{22}^{E}$ é o módulo de elasticidade complexo do PZT e $\rho$ é a densidade do PZT. Separando o deslocamento nos domínios do tempo e espacial e aplicando as condições de contorno, a equação acima pode ser escrita como,

$$
\begin{gathered}
v=\bar{v} e^{i \omega t}=(A \sin (k \cdot y)+B \cos (k \cdot y)) e^{i \omega t} \\
k=\omega^{2} \frac{\rho}{\bar{Y}_{22}^{E}}
\end{gathered}
$$

onde $\omega$ é uma excitação harmônica constante. O deslocamento do atuador PZT, a deformação, o campo de tensão e o campo de deslocamento elétrico podem então ser determinados (Ayres, 1996). O fluxo de corrente no PZT é a taxa de tempo da carga elétrica total entre os dois eletrodos, podendo ser expressa como:

$$
I=\iint_{\sigma} i \omega D_{3} d \sigma
$$

onde $\sigma$ é a área do eletrodo e $D_{3}$ o deslocamento na direção do eixo $\mathrm{z}\left(\mathrm{x}_{3}\right)$. A admitância, inverso da impedância, é $Y=$ $I_{/ V}$ e após algumas substituições, para um PZT retangular, conforme mostrado na figura 2 , pode ser encontrada como (Sun, 1996):

$$
\begin{gathered}
Y(\omega)=i \omega \frac{w_{A} l_{A}}{h_{A}}\left[\bar{\varepsilon}_{33}^{T}-\left(d_{32}\right)^{2} \bar{Y}_{22}^{E}+\right. \\
\left.\frac{Z_{A}(\omega)}{Z_{S}(\omega)+Z_{A}(\omega)}\left(d_{32}\right)^{2} \bar{Y}_{22}^{E} \frac{\tan \left(k \cdot l_{A}\right)}{k \cdot l_{A}}\right]
\end{gathered}
$$


onde $Z_{A}$ é a impedância mecânica do atuador ao longo da direção $y, Z_{S}$ é a impedância mecânica da estrutura e $w_{A}, L_{A}$, $h_{A}$ são a largura, o comprimento e a espessura do elemento PZT, respectivamente.

Uma das vantagens de elementos piezelétricos para aplicações em problemas de controle e localização de falhas resulta na relativa insensibilidade destes materiais quando utilizados abaixo da temperatura de Curie. A variação no tensor de deformação piezelétrico para um material piezocerâmico sob a ação de carga constante é menor que $12 \%$ para temperaturas até $120^{\circ} \mathrm{C}$. Devido ao fato de as deformações sofridas pelo material estarem relacionadas com o valor do campo elétrico a ser introduzido, a estabilidade para a temperatura é crucial em aplicações de controle e detecção de falhas, pois erros provenientes de variações térmicas acarretarão erros na magnitude do controle e poderão fornecer informações errôneas no caso de monitoramento de defeitos estruturais.

A baixa sensibilidade à temperatura dos piezelétricos é uma vantagem sobre os elementos eletro-resistivos, pois estes são altamente dependentes da temperatura. Uma segunda vantagem dos piezelétricos está em sua flexibilidade em uma variedade de aplicações, isto é parcialmente devido a diversidade de materiais que possuem propriedades piezelétricas. Entre os materiais piezelétricos mais utilizados estão os piezocerâmicos e os piezopolímeros, dos quais os PZTs e os PVDFs são respectivos exemplos.

Devido à natureza cerâmica, os PZTs possuem boa rigidez e freqüentemente excede a da estrutura base, o que resulta em uma excelente conversão da energia elétrica em energia mecânica. Isto torna estes elementos eficientes atuadores para uma grande variedade de aplicações. Materiais piezocerâmicos são efetivos sobre uma larga faixa de freqüência, e a dupla propriedade exibida por estes materiais (self-sensing actuators, Dosch et al., 1992) torna extremamente vantajosa sua aplicação para sistemas de controle e de localização de falhas.

Embora vantajosos em muitas aplicações, os piezocerâmicos apresentam dificuldade em assumir moldes com formas complexas devido a alta fragilidade. Alternativamente, os filmes PVDF têm consistência de uma folha plástica e podem ser colados em praticamente qualquer geometria, tem alta sensibilidade e precisão nas medidas e são, portanto, utilizados, principalmente, como sensores. A aplicação direta dos filmes PVDF como atuadores tem sido limitada, pois exibem coeficientes de acoplamento eletromecânico mais baixos que os PZT. No entanto, a resistência dielétrica dos filmes PVDF é maior que a do PZT e eles podem ser expostos a maiores campos elétricos.

O coeficiente de acoplamento eletromecânico garante a conversão efetiva de energia em um sistema de interação linear.
Neste trabalho, o coeficiente de acoplamento eletromecânico (freqüentemente denominado fator de acoplamento) do transdutor piezelétrico é considerado. Para se determinar o coeficiente de acoplamento, a informação da orientação do cristal e o arranjo de entrada e saída são requeridos, em outras palavras, o coeficiente é definido para o respectivo modo de vibração no limite estático. O coeficiente de acoplamento $k$ é medido em percentagem para a maioria dos casos, porém ele tem maior significado quando utilizado ao quadrado, $k^{2}$, o qual corresponde à razão de energia.

Por apresentarem um comportamento aproximadamente linear sob baixos campos elétricos, relativos a insensibilidade à variação de temperatura e flexibilidade como sensores e atuadores, os elementos piezelétricos são mais freqüentemente utilizados em aplicações aeroespaciais e automotivas. Os sensores e atuadores piezocerâmicos, são leves e podem ser colados a uma variedade de estruturas. A maioria das pesquisas atuais considera o efeito do acoplamento eletromecânico do PZT com a estrutura base desprezível. No entanto, para técnicas de ajuste de modelos ou aplicações envolvendo localização de falhas em estruturas leves e específicas este efeito deve ser considerado (Lopes Jr et al., 2000).

\section{OTIMIZAÇÃO POR NUVEM DE PARTÍ- CULAS - PSO}

A PSO foi desenvolvida inicialmente por Kennedy e Eberhart (1995) baseada nos estudos do sócio-biologista Edward Osborne Wilson (Wilson, 1971; Wilson, 1995). A PSO constitui uma técnica da inteligência coletiva baseada em uma população de soluções e transições aleatórias (Brandstätter e Baumgartner, 2002). A PSO apresenta características similares as técnicas da computação evolutiva, que são baseadas em uma população de soluções. Entretanto, a PSO é motivada pela simulação de comportamento social e cooperação entre agentes em vez da sobrevivência do indivíduo mais apto como nos algoritmos evolutivos. Na PSO, cada solução candidata (denominada de partícula) possui associada uma velocidade. A velocidade é ajustada através de uma equação de atualização que considera a experiência da partícula correspondente e a experiência das outras partículas presentes na população (Shi e Eberhart, 1998, 1999).

O conceito da PSO consiste de, a cada passo iterativo, mudar a velocidade de cada partícula em direção as localizações do pbest (melhor posição) e do gbest (melhor partícula). A rapidez do procedimento de busca é ponderada através de um termo gerado de forma aleatória, sendo este vinculado de forma separada as localizações do pbest $\mathrm{e}$ do gbest. O procedimento para implementação da PSO é regido pelas seguintes etapas: 
(i) iniciar uma população (matriz) de partículas, com posições e velocidades em um espaço de problema $n$ dimensional, aleatoriamente com distribuição uniforme;

(ii) para cada partículas, avaliar a função de aptidão (função objetivo);

(iii) comparar a avaliação da função de aptidão da partícula com o pbest da partícula. Se o valor corrente é melhor que pbest, então o valor de pbest passa a ser igual ao valor da função de aptidão da partícula, e a localização do pbest passa a ser igual a localização atual no espaço $n$ dimensional;

(iv) comparar a avaliação da função de aptidão com o prévio melhor valor de aptidão da população. Se o valor atual é melhor que o gbest, atualizar o valor de gbest para o índice e valor da partícula atual;

(v) modificar a velocidade e a posição da partícula de acordo com as equações (10) e (11), respectivamente:

$$
\begin{gathered}
v_{i}=w \cdot v_{i}+c_{1} \cdot u d \cdot\left(p_{i}-x_{i}\right)+c_{2} \cdot U d \cdot\left(p_{g}-x_{i}\right) \\
x_{i}=\left(x_{i}+\Delta t \cdot v_{i}\right)
\end{gathered}
$$

onde $\Delta t$ é igual a 1.

(vi) ir para a etapa (ii) até que um critério de parada seja encontrado, usualmente um valor de erro pré-definido ou um número máximo de iterações (gerações).

As notações usadas são: $x_{i}=\left[x_{i 1}, x_{i 2}, \ldots, x_{i n}\right]^{\mathrm{T}}$ armazena a posição da $i$-ésima partícula, $v_{i}=\left[v_{i 1}, v_{i 2}, \ldots, v_{i n}\right]^{\mathrm{T}}$ armazena a velocidade da $i$-ésima partícula e $p_{i}=$ $\left[p_{i 1}, p_{i 2}, \ldots, p_{i n}\right]^{\mathrm{T}}$ representa a posição do melhor valor de aptidão da $i$-ésima partícula. O índice $g$ representa o índice da melhor particular entre todas as partículas do grupo. A variável $w$ é a ponderação de inércia, $c_{1}$ e $c_{2}$ são constantes positivas; $u d$ e $U d$ são duas funções para geração de números aleatórios com distribuição uniforme no intervalo $[0,1]$, respectivamente. O tamanho da população é selecionado dependendo do problema.

As velocidades das partículas em cada dimensão são limitadas a um valor máximo de velocidade, $V_{\max }$. O $V_{\max }$ é importante, pois determina a resolução que a região próxima às soluções atuais são procuradas. Se $V_{\max }$ é alto, a PSO facilita a busca global, enquanto um valor $V_{\max }$ pequeno enfatiza as buscas locais. A primeira parte na equação (10) é um termo de momento da partícula. A ponderação de inércia $w$ representa o grau de momento da partícula. A segunda parte consiste da parte "cognitiva", que representa o "conhecimento" independente da partícula. A terceira parte é a "social”, que representa a colaboração entre as partículas.

As constantes $c_{1}$ e $c_{2}$ representam a ponderação das partes de "cognição" e "social" que influenciam cada partícula em direção a pbest e a gbest. Estes parâmetros são usualmente ajustados por heurísticas de tentativa e erro. Neste contexto, a literatura que aborda o ajuste das constantes $c_{1} \mathrm{e}$ $c_{2}$ é extensa (Kennedy et al., 2001; Carlisle e Doizier, 2001; Eberhart e Shi, 2001; Hu e Eberhart, 2001; Clerc e Kennedy, 2002; Robinson e Samii, 2004; Ratnaweera et al., 2004; Van den Bergh e Engelbrecht, 2006).

Segundo Ratnaweera et al. (2004), a redução da componente cognitiva e aumento da componente social durante o início da otimização pelo PSO pode ser uma alternativa viável, pois um valor maior de componente cognitiva $c_{1}$ e pequeno valor da componente social $c_{2}$ no início da otimização incentivam as partículas a moverem-se pelo espaço de busca em vez de moverem-se em direção a melhor partícula da população. Em contra partida, um pequeno $c_{1}$ e um grande valor de $c_{2}$ ao final da otimização permite as partículas convergirem para um ótimo local no final da otimização.

No entanto, apesar de diversas heurísticas terem sido desenvolvidas, nos últimos anos, para melhorar o desempenho e escolha adequada de parâmetros para o algoritmo PSO (Shi e Eberhart, 1998; Shi e Eberhart, 1999; Kennedy et al., 2001; Clerc e Kennedy, 2002; Pan et al., 2006) ainda não foi apresentada uma proposta que seja comprovadamente superior as outras apresentadas na literatura. Neste contexto, devese mencionar que várias abordagens da literatura estão objetivando a prova de convergência do PSO sob determinadas condições de projeto de $w, c_{1}$ e $c_{2}$, a citar os trabalhos de Clerc e Kennedy (2002), Trelea (2003), Emara e Fattah (2004), Van den Bergh e Engelbrecht (2006) e Kadirkamanathan et al. (2006).

\subsection{Abordagens de PSO usando distribui- ção Gaussiana e de Cauchy}

A utilização de uma distribuição de probabilidade uniforme para gerar números aleatórios para atualizar a velocidade é uma abordagem clássica em PSO. Entretanto, a utilização de outras distribuições de probabilidade em pode melhorar a habilidade de ajuste fino na busca pelo PSO ou mesmo para escapar de ótimos locais.

$\mathrm{Na}$ literatura tem sido proposta a utilização de distribuições de probabilidade Gaussiana e Cauchy para geração de números aleatórios para atualização da equação da velocidade do PSO, estas inspiradas nos estudos de operadores de mutação em algoritmos de programação evolutiva rápida (fast 
evolutionary programming) (Yao e Liu, 1996; Chellapilla, 1998). Neste contexto, alguns trabalhos recentes sobre PSO têm abordado este assunto. Por exemplo, os trabalhos de Miranda e Fonseca (2002), Wei et al. (2002), Secrest e Lamont (2003), Kennedy (2003), Higashi e Iba (2003), Stacey et al. (2003), Esquivel e Coello (2003), Coelho e Krohling (2003) e Krohling (2004).

As abordagens de PSO propostas e avaliadas, neste artigo, são baseadas nos estudos de Coelho e Krohling (2003) e Krohling (2004). Esta abordagem visa modificar a equação (10) do PSO convencional - PSO do tipo 1 - para utilizála com diferentes combinações das distribuições uniforme, Gaussiana ou de Cauchy.

A utilização de distribuição de Cauchy em algoritmos evolutivos pode ser útil para evitar mínimos locais quando o espaço de busca é pequeno. Enquanto, a distribuição Gaussiana (normal) pode prover uma convergência mais rápida em buscas locais quando o espaço de busca é grande. As abordagens, para modificação da equação (10) do PSO convencional, propostas e avaliadas são:

Tipo 2: Utilização de uma função com distribuição de Cauchy modificada, $c d$, para geração de números aleatórios no intervalo $[0,1]$ da parte "cognitiva":

$$
v_{i}=w \cdot v_{i}+c_{1} \cdot c d \cdot\left(p_{i}-x_{i}\right)+c_{2} \cdot U d \cdot\left(p_{g}-x_{i}\right)
$$

Tipo 3: Utilização de uma função com distribuição de Cauchy modificada, $\mathrm{Cd}$, para geração de números aleatórios no intervalo $[0,1]$ da parte "social":

$$
v_{i}=w \cdot v_{i}+c_{1} \cdot u d \cdot\left(p_{i}-x_{i}\right)+c_{2} \cdot C d \cdot\left(p_{g}-x_{i}\right)
$$

Tipo 4: Utilização de uma função com distribuição de Cauchy modificada, $c d$ e $C d$, para geração de números aleatórios no intervalo $[0,1]$, tanto na parte "cognitiva" quanto na "social":

$$
v_{i}=w \cdot v_{i}+c_{1} \cdot c d \cdot\left(p_{i}-x_{i}\right)+c_{2} \cdot C d \cdot\left(p_{g}-x_{i}\right)
$$

Tipo 5: Utilização de uma função com distribuição de Gauss (normal), $g d$, para geração de números aleatórios no intervalo $[0,1]$ na parte "cognitiva":

$$
v_{i}=w \cdot v_{i}+c_{1} \cdot g d \cdot\left(p_{i}-x_{i}\right)+c_{2} \cdot U d \cdot\left(p_{g}-x_{i}\right)
$$

Tipo 6: Utilização de uma função com distribuição de Gauss (normal), $G d$, para geração de números aleatórios no intervalo $[0,1]$ na parte "social":

$$
v_{i}=w \cdot v_{i}+c_{1} \cdot u d \cdot\left(p_{i}-x_{i}\right)+c_{2} \cdot G d \cdot\left(p_{g}-x_{i}\right)
$$

Tipo 7: Utilização de uma função com distribuição de Gauss (normal), $g d$ e $G d$, para geração de números aleatórios no intervalo $[0,1]$, tanto na parte "cognitiva" quanto na parte "social":

$$
v_{i}=w \cdot v_{i}+c_{1} \cdot g d \cdot\left(p_{i}-x_{i}\right)+c_{2} \cdot G d \cdot\left(p_{g}-x_{i}\right)
$$

Tipo 8: Utilização de uma função com $g d$ na parte "cognitiva" e $C d$ na parte "social":

$$
v_{i}=w \cdot v_{i}+c_{1} \cdot g d \cdot\left(p_{i}-x_{i}\right)+c_{2} \cdot C d \cdot\left(p_{g}-x_{i}\right)
$$

Tipo 9: Utilização de uma função com $G d$ na parte "social" e $c d$ na parte "cognitiva":

$$
v_{i}=w \cdot v_{i}+c_{1} \cdot c d \cdot\left(p_{i}-x_{i}\right)+c_{2} \cdot G d \cdot\left(p_{g}-x_{i}\right)
$$

\section{METODOLOGIA DE ANÁLISE}

A proposta deste trabalho é a identificação de danos em duas fases distintas. Na primeira etapa se determina a localização da falha através do método de impedância elétrica. Este método é baseado em altas faixas de frequiência e modos locais e, portanto, a área de influência de cada atuador é pequena e define com boa exatidão a região da localização da falha. É importante notar que este método não é capaz de fornecer a severidade da falha. A segunda parte desta metodologia fornece informações quantitativas da falha, através de um método de otimização.

O problema direto, que consiste na determinação da variação das propriedades modais em função das variações físicas da estrutura, tem solução única. No entanto, a caracterização de falhas, que é um problema inverso, não apresenta solução única. Qualquer método de otimização que pretenda ajustar o modelo terá grande chance de fracassar para sistemas com nível médio de complexidade ou maior. Existem vários métodos de redução dos modelos ou de escolha das variáveis que serão utilizadas no ajuste. Entre os mais utilizados, pode-se citar o método de sensibilidade; no entanto, a falha pode ocorrer em posições onde a variação daqueles parâmetros apresenta baixa sensibilidade. 


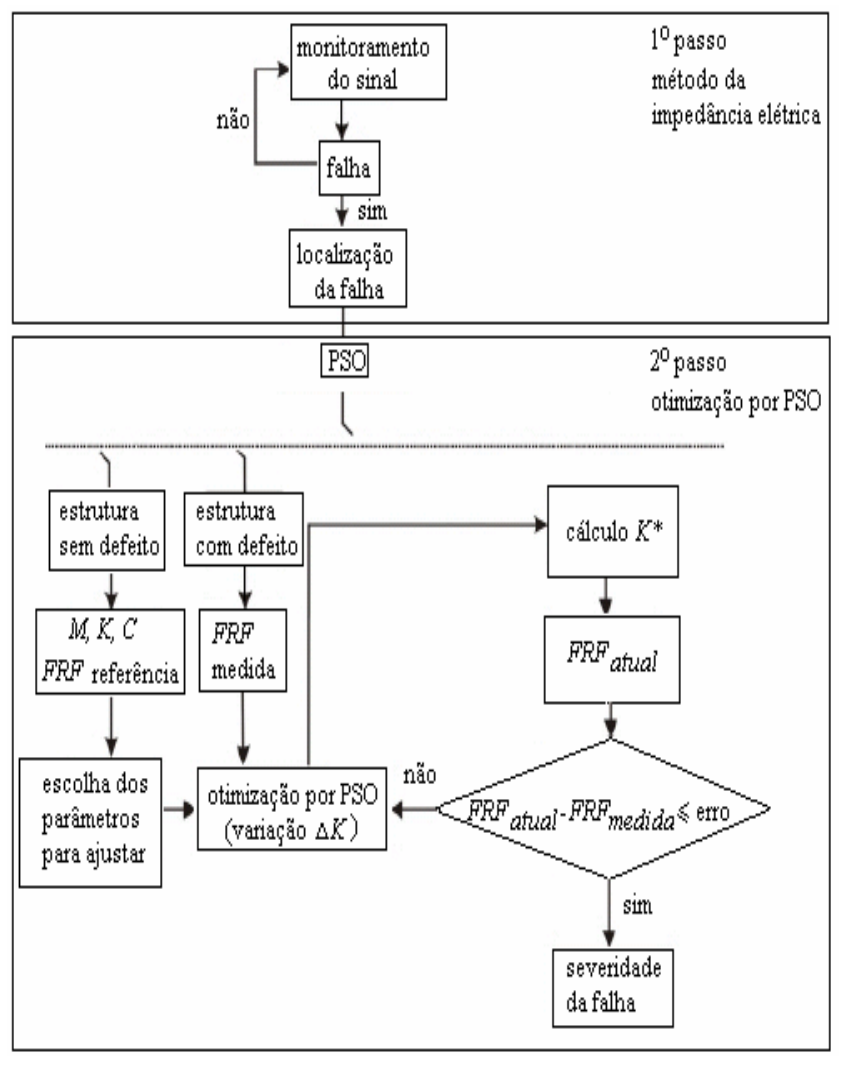

Figura 3: Fluxograma esquemático da metodologia proposta.

A principal vantagem da proposta híbrida apresentada neste trabalho é que o método de impedância elétrica define com exatidão a localização da falha e com isto é possível reduzir o número de variáveis que serão utilizadas no processo de otimização. O método de otimização empregado é baseado em modelo matemático, portanto, é necessário que o modelo inicial da estrutura seja confiável. A figura 3 mostra um fluxograma da metodologia proposta, onde $M$ representa a matriz de massa, $K$ a matriz de rigidez e $C$ a matriz de amortecimento.

A escolha dos parâmetros, que serão utilizados para quantificar a falha, é realizada após a localização da região da falha. Após a definição destes parâmetros, estima-se a variação ocorrida na estrutura em função da falha, obtendo novos valores para $M^{*}, K^{*}$ e $C^{*}$. A $F R F_{\text {atual }}$, obtida com estes novos valores, é, então, ajustada a $F R F_{\text {medida }}$ (situação com defeito) através da técnica de otimização utilizando PSO. Quando a diferença entre estas curvas é menor que um valor especificado o processo termina e a diferença entre as matrizes do sistema sem falha, $M, K$ e $C$, e das matrizes $M^{*}, K^{*} \mathrm{e} C^{*}$ fornece a severidade do defeito.

A otimização foi realizada utilizando a PSO descrita na seção 3. No exemplo mostrado a seguir as matrizes de massa,
$M$, e de rigidez, $K$, foram determinadas através do método de Elementos Finitos. A matriz de amortecimento, $C$, é considerada proporcional a massa e a rigidez. Para a estrutura com defeito foi considerada apenas variação na rigidez $K^{*}$.

A falha métrica foi usada neste trabalho como um índice para monitorar a ocorrência de falhas e, é definida como a soma das diferenças quadráticas das mudanças de impedância para cada frequiência. Este índice simplifica a interpretação das variações de impedância mecânica da estrutura (Lopes Jr $e t$ al., 2000). O monitoramento de cada PZT é feito separadamente, isto é, cada PZT é continuamente monitorado na faixa de frequiência especificada. Para cada PZT, deve-se definir um valor mínimo, acima do qual é uma indicação de falha naquela região. As condições de operação e variações de temperatura podem mudar as características dinâmicas da estrutura. O valor mínimo é definido por tentativa e erro, ou conhecimento da dinâmica estrutural do sistema. A falha métrica, $M$, é dada por:

$$
M=\sum_{i=1}^{n}\left[Z_{i, 1}-Z_{i, 2}\right]^{2}
$$

em que $Z_{i, 1}$ é a impedância medida na estrutura intacta; $Z_{i, 2}$ é a impedância medida na estrutura em condições normais de operação e $i$ é o número de pontos utilizados na aquisição do sinal .

\section{RESULTADOS OBTIDOS}

As medidas experimentais de impedância elétrica foram realizadas com o analisador de impedância HP 4192A. As curvas de impedância foram avaliadas através do valor da falha métrica. Umas das vantagens em se usar a técnica de impedância elétrica é a possibilidade de identificação de falhas simultâneas, em diferentes estágios de evolução.

Para comprovar a capacidade do PSO foram realizados testes para quantificar as falhas estruturais. Nos testes realizados foram analisadas duas situações de falhas, que consistem em cortes transversais a viga, com largura $b$ e profundidade $a$. As figuras 4 e 5 mostram a estrutura analisada na condição livre-livre e medidas de impedância elétrica para diferentes profundidades de falhas na posição 2 , medidas com o PZT número 2 , respectivamente.

O método de quantificação de falhas consiste em minimizar uma função de custo (função objetivo), que possa representar diferenças, em alguma norma, entre as respostas medida e numérica. A função adotada neste trabalho para caracterizar a falha pode ser matematicamente expressa como: 


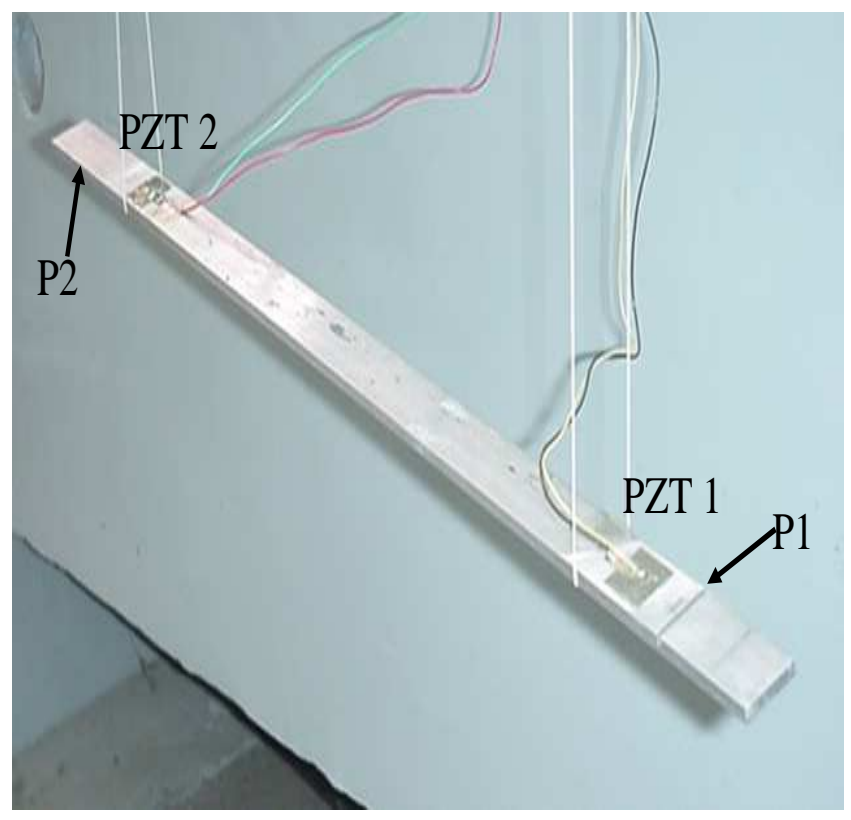

Figura 4: Estrutura utilizada, mostrando a situação de falhas nas posições $\mathrm{P} 1$ e $\mathrm{P} 2$.

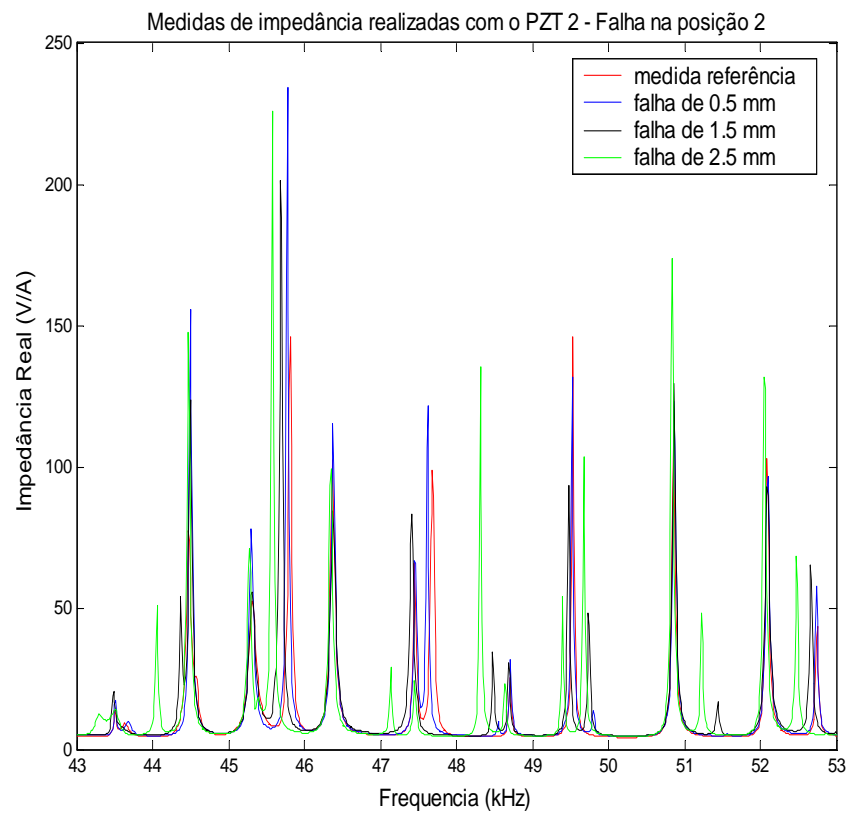

Figura 5: Medidas de impedância elétrica realizadas com o PZT 2.

$$
\begin{gathered}
f_{m}=f_{\text {medida }}(1: 7) \\
f_{a j}=f_{\text {atual }}(1: 7)
\end{gathered}
$$

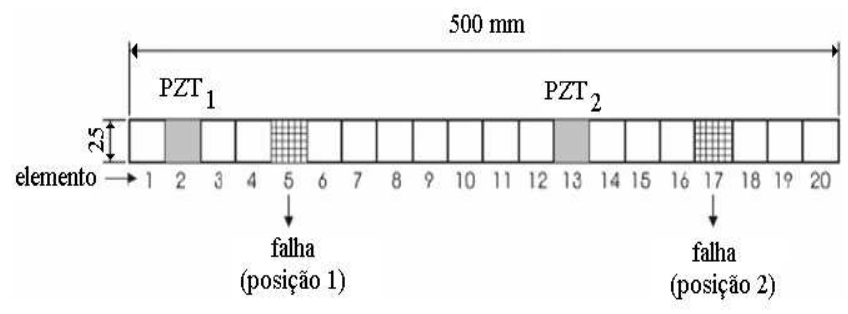

Figura 6: Posição das falhas e dos PZTs na estrutura analisada.

$$
F x e=\sum_{i=1}^{7}\left|f_{m}-f_{a j}\right|
$$

onde $f_{\text {medida }}$ e $f_{\text {atual }}$ são os valores das sete primeiras frequiências naturais, obtidas segundo a metodologia apresentada na figura 3. O valor Fxe considera a somatória dos erros das sete primeiras frequiências naturais. A função de aptidão (fitness function) usada na PSO visa a maximização do valor, portanto, se utiliza o inverso da equação (23).

A figura 6 mostra um desenho esquemático da estrutura analisada. A viga é feita de alumínio com dimensões 500, 25 e $5 \mathrm{~mm}$ de comprimento, largura e espessura, respectivamente. A viga é engastada em uma extremidade e livre na outra. Para a modelagem em elementos finitos se considerou o elemento do tipo Beam e o programa foi implementado no software Matlab, da empresa MathWorks. A estrutura foi modelada com 21 nós e foram considerados 2 graus de liberdade por nó (translação em $y$ e rotação em $z$ ). Na primeira situação de dano é considerada uma falha na região do PZT 1 (ou seja, falha no elemento 5 da estrutura). Na segunda situação de dano é considerada uma outra falha na estrutura no elemento 17 (região do PZT 2), caracterizando, portanto, uma situação de falhas simultâneas.

As propriedades do material piezelétrico são baseadas no material designado por PSI-5A-S4 (Piezo Systems, Inc.) e são dadas na tabela 1 .

Tabela 1: Propriedades do material PZT.

\begin{tabular}{|l|l|}
\hline$E_{P}=63 \mathrm{GPa}$ & $\rho_{P}=7650 \mathrm{~kg} / \mathrm{m}^{3}$ \\
\hline$d_{31}=-190 \times 10^{-12} \mathrm{~m} / \mathrm{V}$ & $d_{33}=-190 \times 10^{-12} \mathrm{~m} / \mathrm{V}$ \\
\hline$c_{11}=1,07 \times 10^{11} \mathrm{~N} / \mathrm{m}^{2}$ & $K^{T}=1800$ \\
\hline$E_{P}=30,705 \mathrm{~N} \cdot \mathrm{m} / \mathrm{V} \cdot \mathrm{m}^{2}$ & $\varepsilon_{33}^{S}=7,33 \times 10^{-9} \mathrm{~F} / \mathrm{m}$ \\
\hline
\end{tabular}

$\mathrm{Na}$ estrutura especificada acima, foram realizados diferentes testes. As falhas foram implementadas através de sucessivos acréscimos na profundidade do corte. Os resultados podem ser avaliados através de dois estudos de caso (observando- 
se a configuração apresentada na figura 5). No primeiro se considerou uma redução de $20 \%$ no momento de inércia do elemento 5. No segundo caso se considerou uma redução no momento de inércia de $20 \%$ no elemento 5 e de $15 \%$ no elemento 17, ou seja, a ocorrência de falhas simultâneas. Esta situação corresponde ao maior dano que foi introduzido na estrutura. No entanto, destaca-se que através do primeiro passo da metodologia foi possível detectar a falha desde seu estagio inicial.

\subsection{Estudo de caso 1: Dano no elemento 5}

\section{Caso 1(a): Otimização do elemento 5}

Neste caso se considera que a primeira etapa da metodologia fornece a posição exata da falha. Portanto, o programa ajusta apenas a rigidez do elemento 5. A figura 7 mostra as curvas das FRFs para as situações sem falha e com falha. Nesta figura, o zoom na região entre 400 e $1100 \mathrm{~Hz}$ mostra a pequena variação das curvas. O objetivo da PSO é a partir da curva sem falha (em azul) ajustar à curva com falha (em vermelho) e assim, através das diferenças entre matrizes, quantificar a falha presente na estrutura.

O caso 1(a) é uma situação ideal e de complexidade relativamente simples, pois para a implementação na PSO a quantificação da falha pode ser comparada com um problema de otimização com apenas uma variável. Para claridade do texto, os resultados deste caso não serão apresentados.

\section{Caso 1(b): Otimização das regiões 4, 5 e 6}

Neste exemplo se verifica a capacidade da PSO quantificar a falha quando a técnica da impedância elétrica localiza uma região de falha, ou seja, a técnica informa que a falha está presente e localizada numa região envolvendo os elementos 4, 5 e 6 . Neste caso, os valores ideais a serem alcançados pela PSO são:

- $I^{4}$ : momento de inércia do elemento 4 (valor ideal $1,000)$;

- $I^{5}$ : momento de inércia do elemento 5 (valor ideal $0,800)$;

- $I^{6}$ : momento de inércia do elemento 6 (valor ideal 1,000).

Nas tabelas 2 a 5 é apresentada uma análise estatística do desempenho das abordagens de PSO para 30 experimentos. As tabelas 2 a 5 apresentam, também, o valor médio, o valor mínimo, o valor máximo e desvio padrão destes resultados. Os parâmetros da PSO utilizados para obtenção destes resultados foram:

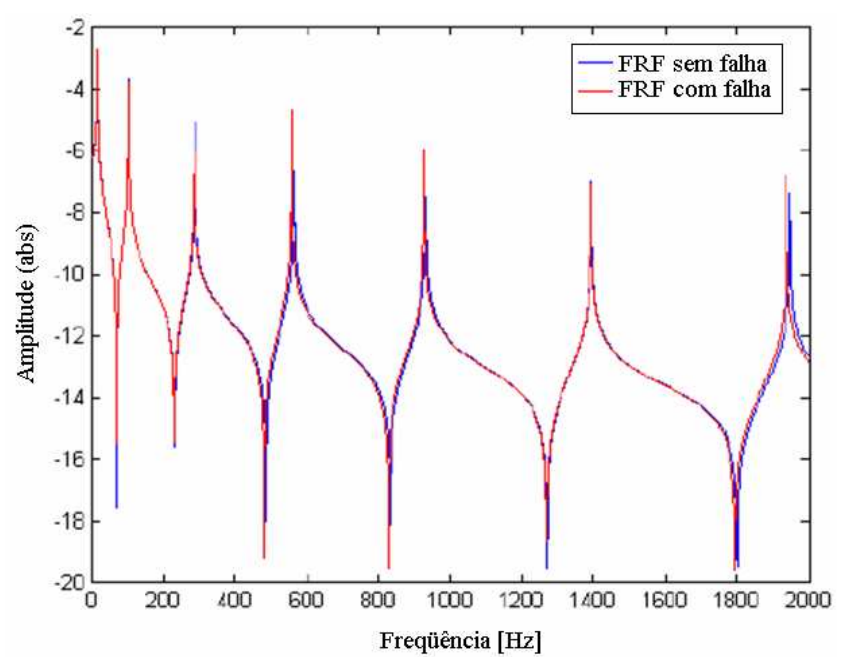

(a)

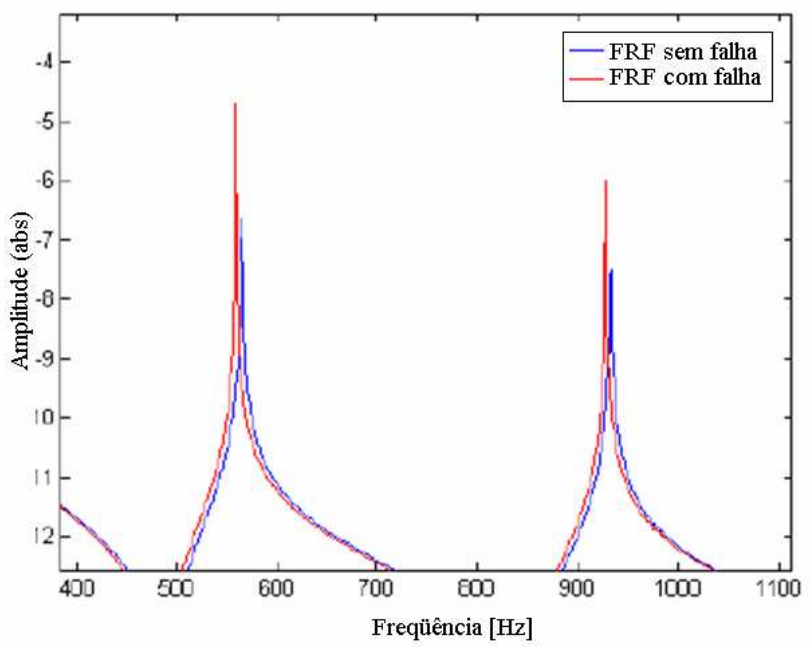

(b)

Figura 7: (a) FRFs da estrutura na situação sem falha e com falha; (b) zoom na faixa de freqüências de 400 a $1100 \mathrm{~Hz}$.

- tamanho da população: 30 partículas;

- espaço de busca das variáveis de momento de inércia $I^{4}, I^{5}, I^{6}\left(\mathrm{~m}^{4}\right): 0<I^{4} \leq 2,0<I^{5} \leq 2$ e $0<I^{6} \leq 2$;

- $V_{\max }: 20 \%$ do espaço de busca de cada variável;

- número máximo de gerações (critério de parada): 100 gerações.

Foram realizados testes com as seguintes configurações de PSO:

(i) $c_{1}=c_{2}=2,0$ (valor constante);

(ii) $c_{1}$ e $c_{2}$ variantes no tempo com valores regidos pelas seguintes equações (Ratnaweera et al., 2004): 


$$
\begin{aligned}
& c_{1}=\left(c_{1 f}-c_{1 i}\right) \frac{g}{G}+c_{1 i} \\
& c_{2}=\left(c_{2 f}-c_{2 i}\right) \frac{g}{G}+c_{2 i}
\end{aligned}
$$

onde $g$ é geração (iteração) atual, $G$ é o número máximo de gerações (critério de parada); adotou-se a configuração proposta por Ratnaweera et al. (2004) com $c_{1 i}=$ $2,5, c_{1 f}=0,5, c_{2 i}=0,5$ e $c_{2 f}=2,5$.

(iii) fator de inércia com decréscimo linear,

$$
w=\frac{G-g}{G}\left(w_{i}-w_{f}\right)+w_{f}
$$

onde o valor inicial de $w$ é $w_{i}=0,9$ e valor final de $w$ é $w_{f}=0,5$ (Kennedy et al., 2001).

(iv) nova proposta de fator de inércia com decréscimo regido pela parte real de um sinal exponencial complexo,

$$
w=R e[\exp (-0,5 g / G)+\cos (20 g)]
$$

onde o valor do co-seno é dado em radianos. Neste caso, o objetivo é enfatizar buscas globais pela PSO.

Nota-se que o PSO(8) apresentou melhor convergência dos resultados (média da função objetivo) nas tabelas 3 e 5 . Contudo, o PSO(2), PSO(8), PSO(6) e PSO (2) obtiveram o melhor resultado (mínimo da função objetivo) para as abordagens das tabelas 2, 3, 4 e 5, respectivamente. Neste caso, os resultados de $I^{4}, I^{5} \mathrm{e} I^{6}$ foram muito próximos dos valores ideais, pois $I^{4}=0,9998, I^{5}=0,8000$ e $I^{6}=0,9999$.

Os algoritmos de PSO foram testados usando Matlab $5.2 \mathrm{em}$ um computador com processador AMD Athon $1,10 \mathrm{GHz}$ e memória RAM de 112 MB. O tempo médio, para a realização de 30 experimentos, foi de aproximadamente 56 segundos e 15 minutos para os caso 1 (b).

\subsection{Estudo de caso 2: Falhas simultâ- neas nos elementos 5 e 17}

Neste estudo de caso são testadas as falhas simultâneas nos elementos 5 e 17. As situações de falha simultânea na estrutura foram: alteração de $20 \%$ no momento de inércia do elemento 5 e $15 \%$ de alteração no momento de inércia do elemento 17. A figura 8 mostra as FRFs para os casos sem falha (curva em azul) e com falhas simultâneas (curva em vermelho).

Neste caso, os valores ideais a serem alcançados pela PSO são:

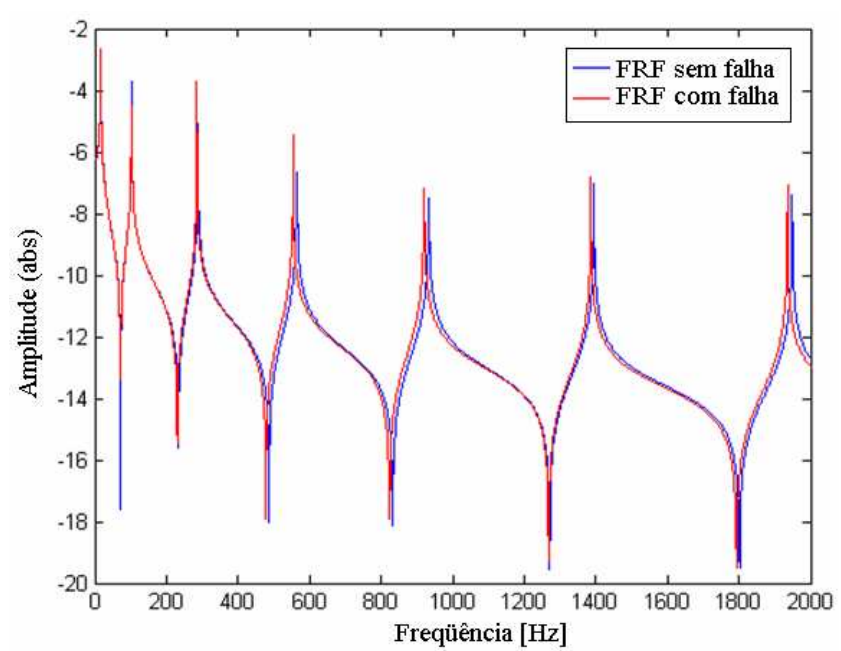

(a)

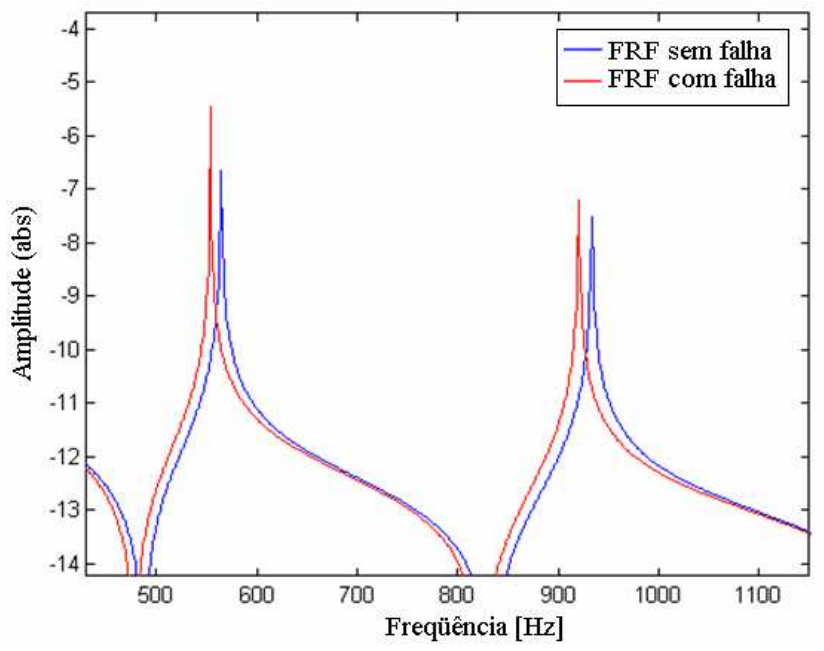

(b)

Figura 8: (a) $F R F s$ da estrutura na situação sem falha e com falha; (b) zoom na faixa de freqüências de 400 a $1100 \mathrm{~Hz}$.

- $I^{4}$ : momento de inércia do elemento 4 (valor ideal $1,000)$

- $I^{5}$ : momento de inércia do elemento 5 (valor ideal $0,800)$;

- $I^{16}$ : momento de inércia do elemento 16 (valor ideal $1,000)$

- $I^{17}$ : momento de inércia do elemento 17 (valor ideal $0,850)$.

Nas tabelas 6 a 9 é apresentada uma análise estatística (valor médio, valor mínimo, valor máximo e desvio padrão) do desempenho das abordagens de PSO para 30 experimentos. Os parâmetros da PSO utilizados para obtenção destes resultados foram: 
- tamanho da população: 30 partículas;

- espaço de busca das variáveis de momento de inércia $I^{4}, I^{5}, I^{16}, I^{17}\left(\mathrm{~m}^{4}\right): 0<I^{4} \leq 2,0<I^{5} \leq 2,0<$ $I^{16} \leq 2$ e $0<I^{17} \leq 2$;

- $V_{\max }: 20 \%$ do espaço de busca de cada variável;

- número máximo de gerações (critério de parada): 100 gerações.

No caso 2 foram utilizadas as mesmas configurações de $c_{1}, c_{2}$ e $w$ do caso 1(b). Nota-se pelos resultados das tabelas 6 a 9 , que o menor valor de função objetivo foi obtida com o PSO(5) com 15,6220 e valores de $I^{4}=1,0000, I^{5}=0,7994$, $I^{16}=1,0013$ e $I^{17}=0,8491$. Estes valores estão próximos os valores ideais mencionados.

Os algoritmos de PSO foram testados no mesmo computador do caso 1(b). Neste caso 2, o tempo médio, para a realização de 30 experimentos, foi de aproximadamente 104 segundos.

Nota-se pelas tabelas 6 a 9 que os resultados obtidos com as abordagens de PSO apresentados nas tabelas 8 e 9 foram superiores em termos de valores médios de função objetivo com relação aos resultados apresentados nas tabelas 6 e 7 .

\subsection{Estudo comparativo do PSO com ou- tras estratégias de otimização}

Na tabela 10 é apresentado um estudo comparativo de melhor geração de solução para os casos 1 e 2. Neste contexto, foram comparados para mesmo tamanho de população e critério de parada, os melhores resultados obtidos com o PSO deste trabalho e as abordagens clássicas de: (i) algoritmos genéticos (Tebaldi, 2004); (ii) programação evolutiva (Tebaldi et al., 2003) e (iii) estratégia evolutiva com mecanismos de auto-adaptação (Tebaldi et al., 2005).

Nota-se pela tabela 10, que o PSO apresentou melhores resultados em relação aos obtidos pelas outras estratégias de otimização, com destaque para o caso de falha simultânea. Baseado nestes resultados, os autores acreditam que quanto maior o número de variáveis envolvidas no processo, mais vantajosa se torna a aplicação da técnica PSO. Os melhores resultados foram obtidos com a PSO seguidos dos resultados obtidos com a estratégia evolutiva.

\section{DISCUSSÃO E CONCLUSÃO}

A tecnologia de materiais inteligentes se tornou uma área de crescente interesse para o desenvolvimento de estruturas que podem incorporar atuadores e sensores capazes de detectar as falhas. Extensivas investigações têm sido focadas na tecnologia que integra materiais inteligentes no monitoramento das condições de sistemas. Vários estudos têm sido realizados na área de identificação de sistemas mecânicos e há uma tendência de introduzir otimização em sistemas de monitoramento da condição da máquina. Este procedimento permite a automatização do processo e a caracterização on-line de falhas estruturais. O problema de identificação de variáveis ou detecção de falhas em sistemas mecânicos é uma classe de problema inverso e, portanto, não apresenta uma solução única. O problema inverso consiste em determinar as causas baseando-se na observação dos efeitos. Nos problemas inversos os parâmetros de falha (comprimento de trinca e/ou localização) podem ser calculados no domínio da freqüência ou do tempo.

A primeira etapa desta metodologia utiliza o método da impedância elétrica para determinar a localização das falhas. Esta técnica é baseada na aplicação de forças de excitação de pequena amplitude e altas faixas de freqüências. Geralmente, utiliza-se tensão elétrica de 1 Volt no PZT e freqüências maiores que $20 \mathrm{kHz}$. Isto faz com que apenas os modos locais de vibração sejam excitados, definindo uma área de influência para cada PZT. Esta característica torna possível a identificação de falhas incipientes nestas áreas de influência de cada sensor/atuador PZT. Na segunda etapa é realizada a quantificação da severidade das falhas usando PSO. A PSO é baseada em uma população de indivíduos (possíveis soluções do problema) e é motivada pela simulação de comportamento social em vez da sobrevivência do indivíduo mais apto.

Neste artigo a caracterização das falhas foi avaliada em uma estrutura com materiais piezelétricos incorporados. As abordagens de PSO implementadas apresentaram resultados precisos e promissores para os casos estudados neste artigo.

A proposta de aplicação conjunta das técnicas de impedância elétrica e da PSO pode oferecer um critério robusto e eficiente para identificação de danos estruturais. Pois, na primeira etapa desta metodologia, a localização da falha pode ser determinada com exatidão e assim os parâmetros de ajuste são reduzidos drasticamente. As vantagens da PSO aliadas ao pequeno número de variáveis a ajustar nos fazem acreditar na potencialidade do método.

Em trabalhos futuros, deseja-se aplicar outras técnicas de otimização da computação evolutiva e inteligência coletiva, para comparação com a das abordagens de PSO para detecção de falhas em estruturas de maior complexidade.

\section{AGRADECIMENTOS}

Os autores agradecem o apoio financeiro da FAPESP, Fundação de Apoio a Pesquisa do Estado de São Paulo, o editor associado e aos revisores deste trabalho, que contribuíram significativamente através de suas sugestões. 


\section{REFERÊNCIAS}

Ayres, J. W. (1996). Qualitative health monitoring and incipient damage inspection/ evaluation, Master thesis, Virginia Polytechnic Institute and State University - CIMSS, USA.

Banks, H. T.; Inman, D. J.; Leo, D. J.; Wang, Y. (1996). An experimentally validated damage detection theory in smart structures, Journal of Sound and Vibrations, vol. 191, no. 5, pp. 859-880.

Baras, J. S.; Tan, X.; Hovareshti, P; (2003). Decentralized control of autonomous vehicles, Proceedings of the 42nd IEEE Conference on Decision and Control, Maui, HI, USA, pp. 1532-1537.

Brandstätter, B.; Baumgartner, U. (2002). Particle swarm optimization - mass-spring systems analogon, IEEE Transactions on Magnetics, vol. 38, no. 2, pp. 997-1000.

Carlisle, A.; Doizier, G. (2001). An off-the-shelf PSO, Proceedings of the Workshop on Particle Swarm Optimization, Indianapolis, IN, USA.

Chellapilla, K. (1998). Combining mutation operators in evolutionary programming, IEEE Transactions on Evolutionary Computation, vol. 2, no. 3, pp. pp. 91-96.

Chen, J.; Patton, R. J. (1993). Robust model-based fault diagnosis for dynamic systems, Kluwer Academic Publishers, Boston, MA, USA.

Clark, R. L.; Saunders, W. R.; Gibbs, G. P. (1998). Adaptive structures: dynamics and control, John Wiley and Sons, Inc.

Clerc, M.; Kennedy, J. (2002). The particle swarm: explosion, stability, and convergence in a multi-dimensional complex space, IEEE Transactions on Evolutionary Computation, vol. 6, no. 1, pp. 58-73.

Coelho, L. S.; R. A. Krohling (2003). Predictive controller tuning using modified particle swarm optimisation based on Cauchy and Gaussian distributions, Proceedings of the 8th Online World Conference on Soft Computing in Industrial Applications, WSC8, Dortmund, Germany.

de Castro, L. N. (2001). Engenharia imunológica: desenvolvimento e aplicação de ferramentas computacionais inspiradas em sistemas imunológicos artificiais, Tese de doutorado, DCA/UNICAMP, Campinas, SP.

Doebling, S. W.; Farrar, C. R.; Prime, M. B. (1998). A summary review of vibration based damage identification methods, The Shock and Vibration Digest, Thousand Oaks, vol. 30, no. 2, pp. 91-105.
Dorigo, M.; Di Caro, G. (1999). The ant colony optimization meta-heuristic, in D. Corne, M. Dorigo, F. Glover (editors), New ideas in optimization, McGraw-Hill, pp. 11-32.

Dosch, J. J.; Inman, D. J.; Garcia, E. (1992). Self-sensing piezoelectric actuator for collocated control, Journal of Intelligent Material Systems and Structures, vol. 3, no. 1, pp. 166-185.

Eberhart, R. C.; Shi, Y. (2001). Tracking and optimizing dynamic systems with particle swarms, Proceedings of Congress on Evolutionary Computation, Seoul, Korea, pp. 94-97.

Emara, H. M.; Fattah, H. A. A. (2004). Continuous swarm optimization technique with stability analysis, Proceedings of American Control Conference, Boston, MA, USA, pp. 2811-2817.

Esquivel, S. C.; Coello, C. A. C. (2003). On the use of particle swarm optimization with multimodal functions, Proceedings of the IEEE Congress on Evolutionary Computation, Canberra, Australia, pp. 1130-1136.

Fourie, P. C.; Groenwold, A. A. (2002). The particle swarm optimization algorithm in size and shape optimization, Structural and Multidisciplinary Optimization, vol. 23, no. 4, pp. 259-267.

Friswell, M. I.; Penny, J. E. (1997). The practical limits of damage detection and location using vibration data, Proceedings of 11th VPI and SU Symposium on Structural Dynamics and Control, Blacksburg, VA, USA, 1-10.

Friswell, M. I.; Penny, J. E. T.; Garvey, S. D. (1998). A Combined genetic and eigensensitivity algorithm for the location of damage in structures, Computers and Structures, vol. 69 , no. 5 , pp. 547-556.

Gabbert, U.; Berger, H.; Koppe, H.; Cao, X. (1998). On modelling and analysis of piezoelectric adaptive structures by the finite element method, Proceedings of 4th European Conference on Smart Structures, Harrogate, UK, pp. 621-628

He, Y.; Guo, D.; Chu, F. (2001). Using genetic algorithms to detect and configure shaft crack for rotor-bearing system, Computer Methods in Applied Mechanics and Engineering, vol. 190, no. 45, pp. 5895-5906.

Higashi, N.; Iba, H. (2003). Particle swarm optimization with Gaussian mutation, Proceedings of the IEEE Swarm Intelligence Symposium, Indianapolis, IN, USA, pp. 7279. 
Hu, X.; Eberhart, R. C. (2001). Tracking dynamic systems with PSO: where's the cheese?, Proceedings of the Workshop on Particle Swarm Optimization, Indianapolis, IN, USA.

Inman, D. J. (1990). Control/structure interaction: effects of actuator dynamics, AIAA Dynamics Specialist Conference, Washington, DC, USA, pp. 311-321.

Inman, D. J. (1996). Engineering vibration, Prentice Hall, NJ, USA.

Inmam, D.J., Farrar, C.R., Lopes Jr., V. and Steffen Jr. V. (2005). Damage prognosis for Aerospace, Civil and Mechanical Systems, John Wiley and Sons.

Kabeya, K. (1998). Structural health monitoring using multiple piezoelectric sensors and actuators, Master thesis, Virginia Polytechnic Institute and State University - CIMSS, USA.

Kadirkamanathan, V.; Selvarajah, K.; Fleming, P. J. (2006). Stability analysis of the particle dynamics in particle swarm optimizer, IEEE Transactions on Evolutionary Computation, vol. 10, no. 3, pp. 245-255.

Kaminski, P. C. (1997). Um sistema de diagnóstico de falhas estruturais baseado em redes neurais artificiais e freqüências naturais, Tese de livre docência, Escola Politécnica da Universidade de São Paulo, São Paulo, SP.

Kannan, S.; Slochanal, S. M. R.; Subbaraj, P.; Padhy, N. P. (2004). Application of particle swarm optimization technique and its variant to generation expansion planning problem, Electric Power Systems Research, vol. 70, no. 3, pp. 203-210.

Kennedy, J. (2003). Bare bones particle swarms, Proceedings of the IEEE Swarm Intelligence Symposium, Indianapolis, IN, USA, pp. 80-87.

Kennedy, J. F.; Eberhart, R.C.; Shi, Y. (2001). Swarm intelligence. San Francisco, CA, USA: Morgan Kaufmann.

Kennedy, J.; Eberhart, R. C. (1995). Particle swarm optimization, IEEE International Conference on Neural Networks, Perth, Australia, vol. 4, pp. 1942-1948.

Kessler, S. S.; Spearing, S. M.; Atalla, M. J. (2002). In-situ damage detection of composites structures using lamb wave methods. Technical Report EWSHM-2002, Technology Laboratory for Advanced Composites, Department of Aeronautics and Astronautics Massachusetts Institute of Technology.

Krohling, R. A. (2004). Gaussian swarm: a novel particle swarm optimization algorithm, Proceedings of the IEEE Conference on Cybernetics and Intelligent Systems, Singapore, pp. 372-376.
Liu, Y.; Passino, K. M. (2004). Stable social foraging swarms in a noisy environment, IEEE Transactions on Automatic Control, vol. 49, no. 1, pp. 30-44.

Lopes Jr., V.; Park, G.; Cudney, H. H.; Inman, D. J. (2000). Impedance-based structural health monitoring with artificial neural network, Journal of Intelligent Material Systems and Structures, vol. 11, no. 13, pp. 206-214.

Marwala, T.; Chakraverty, S. (2006). Fault classification in structures with incomplete measured data using autoassociative neural networks and genetic algorithm, Current Science, vol. 90, no. 4, pp. 542-548.

Miranda, V.; Fonseca, N. (2002). EPSO - Best of two worlds meta-heuristic applied to power system problems, Proceedings of the IEEE Congress on Evolutionary Computation, Honolulu, HI, USA, pp. 1080-1085.

Müller, S. D.; Marchetto, J.; Airaghi, S.; Koumoutsakos, P. (2002). Optimization based on bacterial chemotaxis, IEEE Transactions on Evolutionary Computation, vol. 6, no. 1, pp. 16-29.

Pan, H.; Wang, L.; Liu, B. (2006). Particle swarm optimization for function optimization in noisy environment, Applied Mathematics and Computation, Elsevier (aceito para publicação).

Patton, R. J.; Uppal, F. J.; Lopez-Toribio, C. J. (2000). Soft computing approaches to fault diagnosis for dynamic systems: a survey, IFAC Symposium SAFEPROCESS 2000, Budapest, Hungary, pp. 298-311.

Ratnaweera, A.; Halgamuge, S.; Watson, H. (2004). Selforganizing hierarchical particle swarm optimizer with time-varying acceleration coefficients, IEEE Transactions on Evolutionary Computation, vol. 8, no. 3, pp. 240-255.

Robinson, J.; Samii, Y. R-. (2004). Particle swarm optimization in electromagnetics, IEEE Transactions on Antennas and Propagation, vol. 52, no. 2, pp. 397-407.

Secrest, B. R.; Lamont, G. B. (2003). Visualizing particle swarm optimization - Gaussian particle swarm optimization, Proceedings of the IEEE Swarm Intelligence Symposium, Indianapolis, IN, USA, pp. 198-204.

Shi, Y.; Eberhart, R. C. (1998). A modified particle swarm optimizer, IEEE International Conference on Evolutionary Computation, Anchorage, AK, USA, pp. 69-73.

Shi, Y.; Eberhart, R. C. (1999). Empirical study of particle swarm optimization, IEEE International Conference on Evolutionary Computation, Washington, DC, USA, pp. 1945-1950. 
Silcox, R. J., Lefebvre, S., Metcalf, V. L., Fuller, C. R. (1992). Evaluation of piezoceramic actuators for control of aircraft interior noise, Proceeding of 14th Aeroacoustic Conference, DGLR/AIAA, Aachen, Germany.

Simões, R. C.; Steffen Jr., V. (2002). Sobre o problema da identificação de falhas em máquinas rotativas, II Congresso Nacional de Engenharia Mecânica, II CONEM, João Pessoa, PB (CD-ROM).

Stacey, A.; Jancic, M.; Grundy, I. (2003). Particle swarm optimization with mutation, Proceedings of the IEEE Congress on Evolutionary Computation, Canberra, Australia, pp. 1425-1430.

Sun, F. (1996). Piezoelectric active sensor and electric impedance approach for structural dynamic measurement, Master thesis, Virginia Polytechnic Institute and State University - CIMSS, USA.

Tebaldi, A. (2004). Detecção de falhas estruturais usando sensores e atuadores piezoelétricos e algoritmos genéticos, Dissertação de mestrado, Pós-Graduação em Engenharia Mecânica, Faculdade de Engenharia, Universidade Estadual Paulista Júlio de Mesquita Filho, Ilha Solteira, SP.

Tebaldi, A.; Coelho, L. S.; Lopes Jr., V. (2003). Identificação de falhas em estruturas inteligentes baseada em otimização através de programação evolutiva, XXIV Iberian Latin-American Congress on Computational Methods in Engineering, XXIV CILAMCE, Ouro Preto, MG.

Tebaldi, A., Coelho, L. S.; Lopes Jr., V. (2005). Detecção de falhas em estruturas inteligentes usando estratégia evolutiva com mecanismos de auto-adaptação, VII Simpósio Brasileiro de Automação Inteligente, São Luís, MA.

Trelea, I. C. (2003). The particle swarm optimization algorithm: convergence analysis and parameter selection, Information Processing Letters, vol. 85, no. 6, pp. 317325 .

Tseng, K. K., Wang, L. (2005). Impedance-based method for nondestrutive damage identification, Journal of Engineering Mechanics - ASCE, vol. 131, no. 1, pp. 58-64.

Van den Bergh, F; Engelbrecht, A. P. (2006). A study of particle swarm optimization particle trajectories, Information Sciences, vol. 176, pp. 937-971.

Xu, J.; Yang, Y.; Soh, C. K. (2004). Electromechanical impedance-based structural health monitoring with evolutionary programming, Journal of Aerospace Engineering, vol. 17, no. 5, pp. 182-193.
Yang, Y.; Soh, C. K.; Xu, J. (2005). An integrated evolutionary programming and impedance-based NDE method, Proceedings of SPIE, vol. 5647, Smart Structures, Devices, and Systems II, S. F. Al-Sarawi (ed.), pp. 154161.

Yao, X.; Liu, Y. (1996). Fast evolutionary programming, Proceedings of 5th Annual Conference on Evolutionary Programming, San Diego, CA, USA, pp. 451-460.

Wei, C.; He, Z.; Zheng, Y.; Pi, W. (2002). Swarm directions embedded in fast evolutionary programming, Proceedings of the IEEE Congress on Evolutionary Computation, Honolulu, HI, USA, pp. 1278-1283.

Wilson, E.O. (1971). The insect societies, Belknap Press of Harvard University Press, Cambridge, MA.

Wilson, E. O. (1995). Naturalist, Warner Books, New York, NY, USA. 
Tabela 2: Resultados de otimização usando PSO $\operatorname{com} c_{1}=c_{2}=2$ (constantes) e decréscimo linear do fator de inércia para o caso 1(b) (30 experimentos).

\begin{tabular}{|c|c|c|c|c|c|c|c|c|c|c|}
\hline \multicolumn{9}{|c|}{ função objetivo } & \multicolumn{3}{|c|}{ gerações para obter o mínimo da função objetivo } \\
\hline PSO & máximo & mínimo & médio & mediana & $\begin{array}{c}\text { desvio } \\
\text { padrão }\end{array}$ & máximo & mínimo & média & mediana & $\begin{array}{c}\text { desvio } \\
\text { padrão }\end{array}$ \\
\hline$(1)$ & 0,7942 & 0,1241 & 0,3709 & 0,3270 & 0,1802 & 98 & 45 & 72 & 72 & 18 \\
\hline$(2)$ & $\mathbf{0 , 3 7 4 5}$ & $\mathbf{0 , 0 9 2 6}$ & 0,2533 & 0,2835 & 0,1050 & 98 & 52 & 77 & 78 & 15 \\
\hline$(3)$ & 0,4582 & 0,1766 & 0,2867 & 0,2628 & 0,0865 & 97 & 55 & 74 & 71 & 13 \\
\hline$(4)$ & 0,3779 & 0,1639 & $\mathbf{0 , 2 4 8 5}$ & 0,2505 & $\mathbf{0 , 0 7 1 7}$ & 97 & $\mathbf{3 2}$ & $\mathbf{6 6}$ & $\mathbf{6 7}$ & 25 \\
\hline$(5)$ & 0,7060 & 0,1699 & 0,3101 & 0,2933 & 0,1552 & 100 & 44 & 79 & 83 & 17 \\
\hline$(6)$ & 0,5079 & 0,1245 & 0,3275 & 0,3292 & 0,1301 & 100 & 65 & 87 & 92 & $\mathbf{1 2}$ \\
\hline$(7)$ & 0,4423 & 0,1551 & 0,2751 & 0,2740 & 0,0898 & $\mathbf{9 4}$ & 40 & 70 & 75 & 16 \\
\hline$(8)$ & 0,4478 & 0,1674 & 0,2872 & 0,2765 & 0,0781 & 98 & 50 & 87 & 90 & 14 \\
\hline$(9)$ & 0,5572 & 0,1312 & 0,2615 & $\mathbf{0 , 2 3 0 8}$ & 0,1194 & $\mathbf{9 4}$ & 58 & 75 & 71 & 14 \\
\hline média & 0,5184 & 0,1450 & 0,2912 & 0,2808 & 0,1129 & 97 & 49 & 76 & 77 & 16 \\
\hline
\end{tabular}

Tabela 3: Resultados de otimização usando PSO $\operatorname{com} c_{1}=c_{2}=2$ (constantes) e decréscimo usando função exponencial e co-seno do fator de inércia para o caso 1(b) (30 experimentos).

\begin{tabular}{|c|c|c|c|c|c|c|c|c|c|c|}
\hline \multicolumn{4}{|c|}{ função objetivo } & \multicolumn{3}{|c|}{ gerações para obter o mínimo da função objetivo } \\
\hline PSO & máximo & mínimo & médio & mediana & $\begin{array}{c}\text { desvio } \\
\text { padrão }\end{array}$ & máximo & mínimo & média & mediana & $\begin{array}{c}\text { desvio } \\
\text { padrão }\end{array}$ \\
\hline$(1)$ & 0,6265 & 0,2141 & 0,3766 & 0,3657 & 0,1354 & 100 & 50 & 80 & 85 & 20 \\
\hline$(2)$ & 0,5241 & 0,1919 & 0,3633 & 0,3470 & $\mathbf{0 , 1 0 7 6}$ & 99 & $\mathbf{2 7}$ & 73 & 75 & 21 \\
\hline$(3)$ & 0,4928 & 0,2243 & 0,3554 & 0,3443 & 0,0804 & 100 & 30 & 71 & 70 & 25 \\
\hline$(4)$ & 0,5931 & 0,1066 & 0,3609 & 0,4076 & 0,1619 & 97 & 49 & 71 & 65 & 17 \\
\hline$(5)$ & 0,5033 & 0,1527 & 0,3067 & 0,3168 & 0,0959 & 88 & 51 & 67 & 63 & $\mathbf{1 4}$ \\
\hline$(6)$ & 0,5871 & 0,1618 & 0,3949 & 0,4202 & 0,1468 & $\mathbf{8 5}$ & 35 & 61 & 60 & 18 \\
\hline$(7)$ & $\mathbf{0 , 4 3 8 1}$ & 0,1968 & 0,3205 & 0,2952 & 0,0816 & 91 & 29 & $\mathbf{5 5}$ & $\mathbf{4 9}$ & 21 \\
\hline$(8)$ & 0,5435 & $\mathbf{0 , 0 6 9 3}$ & $\mathbf{0 , 2 8 1 1}$ & $\mathbf{0 , 2 8 7 9}$ & 0,1522 & 97 & 46 & 76 & 78 & 16 \\
\hline$(9)$ & 0,5739 & 0,1381 & 0,3417 & 0,3197 & 0,1186 & 98 & 33 & 68 & 72 & 21 \\
\hline média & 0,5425 & 0,1617 & 0,3446 & 0,3449 & 0,1200 & 95 & 39 & 69 & 69 & 19 \\
\hline
\end{tabular}

Tabela 4: Resultados de otimização usando PSO $\operatorname{com} c_{1}$ e $c_{2}$ variantes e decréscimo linear do fator de inércia para o caso 1(b) (30 experimentos).

\begin{tabular}{|c|c|c|c|c|c|c|c|c|c|c|}
\hline \multicolumn{4}{|c|}{ função objetivo } & \multicolumn{3}{|c|}{ gerações para obter o mínimo da função objetivo } \\
\hline PSO & máximo & mínimo & médio & mediana & $\begin{array}{c}\text { desvio } \\
\text { padrão }\end{array}$ & máximo & mínimo & média & mediana & $\begin{array}{c}\text { desvio } \\
\text { padrão }\end{array}$ \\
\hline$(1)$ & 0,2936 & 0,0666 & 0,1972 & 0,2068 & 0,0827 & 100 & 54 & 74 & 69 & 17 \\
\hline$(2)$ & 0,4075 & 0,1602 & 0,2579 & 0,2537 & 0,0797 & 100 & 46 & 78 & 79 & 20 \\
\hline$(3)$ & 0,3273 & 0,0593 & 0,1863 & 0,1828 & 0,0809 & 93 & 42 & 77 & 86 & 18 \\
\hline$(4)$ & 0,2937 & 0,0615 & 0,1815 & 0,1905 & 0,0829 & 100 & 57 & 82 & 89 & 15 \\
\hline$(5)$ & 0,4315 & 0,0536 & 0,2517 & 0,2819 & 0,1146 & 99 & 61 & 84 & 85 & 14 \\
\hline$(6)$ & $\mathbf{0 , 2 7 2 1}$ & $\mathbf{0 , 0 4 9 3}$ & $\mathbf{0 , 1 7 3 5}$ & $\mathbf{0 , 1 7 6 6}$ & $\mathbf{0 , 0 6 2 0}$ & 100 & $\mathbf{3 2}$ & 79 & 82 & 19 \\
\hline$(7)$ & 0,3759 & 0,0587 & 0,2581 & 0,2813 & 0,1028 & 100 & 55 & 77 & 77 & 17 \\
\hline$(8)$ & 0,3109 & 0,0820 & 0,1814 & 0,1778 & 0,0637 & $\mathbf{9 0}$ & 50 & 72 & 71 & $\mathbf{1 3}$ \\
\hline$(9)$ & 0,2957 & 0,1391 & 0,2212 & 0,2090 & 0,0518 & 91 & 30 & $\mathbf{6 0}$ & $\mathbf{5 6}$ & 21 \\
\hline média & 0,3342 & 0,0811 & 0,2120 & 0,2178 & 0,0801 & 97 & 47 & 76 & 77 & 17 \\
\hline
\end{tabular}


Tabela 5: Resultados de otimização usando PSO $\operatorname{com} c_{1}$ e $c_{2}$ variantes e decréscimo usando função exponencial e co-seno do fator de inércia para o caso 1(b) (30 experimentos).

\begin{tabular}{|c|c|c|c|c|c|c|c|c|c|c|}
\hline \multicolumn{9}{|c|}{ função objetivo } & \multicolumn{3}{|c|}{ gerações para obter o mínimo da função objetivo } \\
\hline PSO & máximo & mínimo & médio & mediana & $\begin{array}{c}\text { desvio } \\
\text { padrão }\end{array}$ & máximo & mínimo & média & mediana & $\begin{array}{c}\text { desvio } \\
\text { padrão }\end{array}$ \\
\hline$(1)$ & 0,3372 & 0,1135 & 0,2211 & 0,2270 & 0,0657 & 94 & 31 & 65 & 61 & 21 \\
\hline$(2)$ & 0,3343 & $\mathbf{0 , 0 4 1 4}$ & 0,2330 & 0,2545 & 0,0885 & 95 & 36 & 61 & $\mathbf{5 6}$ & 19 \\
\hline$(3)$ & 0,2626 & 0,0481 & 0,1786 & 0,1791 & 0,0638 & 96 & 29 & 64 & 68 & 24 \\
\hline$(4)$ & 0,3053 & 0,0381 & 0,1897 & 0,1999 & 0,0763 & 99 & 36 & 73 & 71 & 22 \\
\hline$(5)$ & 0,4018 & 0,0688 & 0,1938 & 0,1850 & 0,1078 & $\mathbf{8 3}$ & 29 & $\mathbf{5 6}$ & $\mathbf{5 6}$ & 17 \\
\hline$(6)$ & 0,2468 & 0,0976 & 0,1684 & 0,1664 & $\mathbf{0 , 0 4 6 0}$ & 95 & $\mathbf{1 3}$ & 61 & 57 & 26 \\
\hline$(7)$ & 0,3580 & 0,1021 & 0,2403 & 0,2529 & 0,0881 & 97 & 43 & 70 & 59 & 21 \\
\hline$(8)$ & 0,2632 & 0,0573 & $\mathbf{0 , 1 5 7 2}$ & $\mathbf{0 , 1 5 9 3}$ & 0,0710 & 90 & 36 & 65 & 64 & 19 \\
\hline$(9)$ & $\mathbf{0 , 2 4 3 0}$ & 0,0850 & 0,1661 & 0,1710 & 0,0520 & 88 & 38 & 63 & 60 & $\mathbf{1 6}$ \\
\hline média & 0,3058 & 0,0724 & 0,1942 & 0,1995 & 0,0732 & 93 & 32 & 64 & 61 & 21 \\
\hline
\end{tabular}

Tabela 6: Resultados de otimização usando PSO $\operatorname{com} c_{1}=c_{2}=2$ (constantes) e decréscimo linear do fator de inércia para o caso 2 (30 experimentos).

\begin{tabular}{|c|c|c|c|c|c|c|c|c|c|c|}
\hline \multicolumn{4}{|c|}{ função objetivo } & \multicolumn{3}{|c|}{ gerações para obter o mínimo da função objetivo } \\
\hline PSO & máximo & mínimo & médio & mediana & $\begin{array}{c}\text { desvio } \\
\text { padrão }\end{array}$ & máximo & mínimo & média & mediana & $\begin{array}{c}\text { desvio } \\
\text { padrão }\end{array}$ \\
\hline$(1)$ & 193,1899 & 72,5890 & 115,6720 & 112,2666 & 37,1740 & 97 & 67 & 89 & 93 & 10 \\
\hline$(2)$ & 131,2629 & 46,9650 & 87,1996 & 87,6621 & 22,7564 & 97 & 57 & 81 & 85 & 12 \\
\hline$(3)$ & 130,6144 & 52,5654 & 88,6624 & 89,1310 & 24,1712 & $\mathbf{9 0}$ & 62 & $\mathbf{7 5}$ & 75 & 10 \\
\hline$(4)$ & $\mathbf{1 0 2 , 7 6 9 8}$ & $\mathbf{3 1 , 5 1 5 1}$ & $\mathbf{6 6 , 3 7 2 2}$ & $\mathbf{5 4 , 0 1 7 4}$ & 28,1484 & 100 & 57 & 76 & $\mathbf{7 4}$ & 14 \\
\hline$(5)$ & 196,5371 & 44,3626 & 108,8639 & 100,7812 & 50,9243 & 98 & 76 & 86 & 88 & 8 \\
\hline$(6)$ & 183,2158 & 48,9431 & 104,0343 & 112,5530 & 39,0539 & 100 & 86 & 92 & 92 & $\mathbf{4}$ \\
\hline$(7)$ & 189,8996 & 67,3446 & 112,3347 & 109,0274 & 31,2824 & 98 & 56 & 81 & 85 & 14 \\
\hline$(8)$ & 137,2103 & 57,9532 & 82,6189 & 73,2948 & 23,6357 & 95 & $\mathbf{4 9}$ & 86 & 91 & 15 \\
\hline$(9)$ & 124,3716 & 59,4781 & 83,8089 & 78,3118 & $\mathbf{1 9 , 1 6 1 0}$ & 96 & 63 & 83 & 81 & 11 \\
\hline média & 154,3413 & 53,5240 & 94,3963 & 90,7828 & 30,7008 & 97 & 64 & 83 & 85 & 11 \\
\hline
\end{tabular}

Tabela 7: Resultados de otimização usando PSO com $c_{1}=c_{2}=2$ (constantes) e decréscimo usando função exponencial e co-seno do fator de inércia para o caso 2 (30 experimentos).

\begin{tabular}{|c|c|c|c|c|c|c|c|c|c|c|}
\hline \multicolumn{9}{|c|}{ função objetivo } & \multicolumn{3}{|c|}{ gerações para obter o mínimo da função objetivo } \\
\hline PSO & máximo & mínimo & médio & mediana & $\begin{array}{c}\text { desvio } \\
\text { padrão }\end{array}$ & máximo & mínimo & média & mediana & $\begin{array}{c}\text { desvio } \\
\text { padrão }\end{array}$ \\
\hline$(1)$ & 179,1551 & 79,0298 & 116,8952 & 109,4406 & 30,6875 & 96 & 55 & 71 & $\mathbf{6 8}$ & $\mathbf{1 3}$ \\
\hline$(2)$ & 156,0991 & $\mathbf{2 8 , 4 8 9 0}$ & 92,0265 & $\mathbf{8 9 , 1 8 4 4}$ & 33,5242 & 88 & 40 & $\mathbf{7 0}$ & 69 & 16 \\
\hline$(3)$ & 149,9794 & 47,8750 & 105,5845 & 106,9327 & 32,2861 & 100 & 46 & 80 & 89 & 21 \\
\hline$(4)$ & 134,3865 & 44,1244 & $\mathbf{8 6 , 8 5 2 7}$ & 80,0509 & 35,0962 & $\mathbf{9 4}$ & 44 & 74 & 80 & 16 \\
\hline$(5)$ & 164,9183 & 68,5024 & 122,1791 & 116,7635 & 34,0924 & 100 & 52 & 86 & 94 & 16 \\
\hline$(6)$ & 122,0664 & 63,2159 & 91,8170 & 93,3065 & $\mathbf{2 0 , 1 2 3 4}$ & 97 & 46 & 77 & 80 & 18 \\
\hline$(7)$ & 174,7487 & 59,5124 & 124,5881 & 137,1295 & 37,0516 & 97 & 41 & 73 & 75 & 17 \\
\hline$(8)$ & $\mathbf{1 2 1 , 1 7 3 9}$ & 52,6435 & 98,6597 & 107,7126 & 21,9564 & 100 & $\mathbf{3 9}$ & 78 & 83 & 19 \\
\hline$(9)$ & 160,7948 & 52,2079 & 93,9090 & 94,7725 & 34,5735 & 100 & 52 & 76 & 78 & 15 \\
\hline média & 151,4802 & 55,0667 & 103,6124 & 103,9215 & 31,0435 & 97 & 46 & 76 & 80 & 17 \\
\hline
\end{tabular}


Tabela 8: Resultados de otimização usando PSO $\operatorname{com} c_{1}$ e $c_{2}$ variantes e decréscimo linear do fator de inércia para o caso 2 (30 experimentos).

\begin{tabular}{|c|c|c|c|c|c|c|c|c|c|c|}
\hline \multicolumn{4}{|c|}{ função objetivo } & \multicolumn{4}{|c|}{ gerações para obter o mínimo da função objetivo } \\
\hline PSO & máximo & mínimo & médio & mediana & $\begin{array}{l}\text { desvio } \\
\text { padrão }\end{array}$ & máximo & mínimo & média & mediana & $\begin{array}{c}\text { desvio } \\
\text { padrão }\end{array}$ \\
\hline$(1)$ & 96,3871 & 35,5070 & 58,8738 & 54,7828 & 19,3078 & 100 & 72 & 86 & 88 & 10 \\
\hline$(2)$ & 90,3414 & 34,9947 & 63,8929 & 67,1440 & $\mathbf{1 5 , 7 8 2 9}$ & 95 & 41 & 72 & 74 & 18 \\
\hline$(3)$ & 95,4500 & 34,1545 & 58,7978 & 54,7508 & 18,9803 & 96 & 57 & 79 & 80 & 12 \\
\hline$(4)$ & $\mathbf{8 1 , 3 5 6 1}$ & 18,2333 & $\mathbf{5 4 , 6 8 1 1}$ & $\mathbf{5 0 , 2 0 6 1}$ & 19,1993 & 94 & 57 & 78 & 80 & 14 \\
\hline$(5)$ & 119,2573 & $\mathbf{1 5 , 6 2 2 0}$ & 75,4762 & 77,2767 & 27,5274 & 95 & 63 & 82 & 84 & 10 \\
\hline$(6)$ & 89,1132 & 46,3641 & 70,6868 & 69,6558 & 12,5333 & 97 & $\mathbf{3 0}$ & 77 & 83 & 21 \\
\hline$(7)$ & 115,0005 & 27,8445 & 63,2435 & 60,6496 & 22,2095 & 93 & 64 & 80 & 84 & $\mathbf{9}$ \\
\hline$(8)$ & 99,5002 & 36,3270 & 63,7008 & 61,5898 & 22,0445 & 100 & 64 & 85 & 88 & 12 \\
\hline$(9)$ & 94,0783 & 17,4954 & 60,6389 & 63,5748 & 23,5271 & $\mathbf{8 1}$ & 43 & $\mathbf{6 7}$ & $\mathbf{7 1}$ & 14 \\
\hline média & 97,8316 & 29,6158 & 63,3324 & 62,1812 & 20,1236 & 95 & 55 & 78 & 81 & 13 \\
\hline
\end{tabular}

Tabela 9: Resultados de otimização usando PSO $\operatorname{com} c_{1}$ e $c_{2}$ variantes e decréscimo usando função exponencial e co-seno do fator de inércia para o caso 2 (30 experimentos).

\begin{tabular}{|c|c|c|c|c|c|c|c|c|c|c|}
\hline \multicolumn{4}{|c|}{ função objetivo } & \multicolumn{3}{|c|}{ gerações para obter o mínimo da função objetivo } \\
\hline PSO & máximo & mínimo & médio & mediana & $\begin{array}{c}\text { desvio } \\
\text { padrão }\end{array}$ & máximo & mínimo & média & mediana & $\begin{array}{c}\text { desvio } \\
\text { padrão }\end{array}$ \\
\hline$(1)$ & 71,8178 & 53,2847 & 60,3443 & 58,6528 & $\mathbf{5 , 8 4 9 8}$ & 100 & 46 & 70 & 71 & 18 \\
\hline$(2)$ & 84,3854 & 22,5250 & 48,5841 & $\mathbf{4 2 , 0 6 9 7}$ & 22,4900 & 96 & 42 & 69 & 66 & 19 \\
\hline$(3)$ & 70,5101 & 23,9729 & $\mathbf{4 4 , 8 3 4 4}$ & 44,0335 & 15,5675 & $\mathbf{9 0}$ & 48 & 67 & 68 & $\mathbf{1 3}$ \\
\hline$(4)$ & $\mathbf{6 1 , 2 6 1 9}$ & $\mathbf{1 5 , 8 2 5 4}$ & 45,8977 & 46,4360 & 12,8982 & 99 & 38 & 73 & 77 & 19 \\
\hline$(5)$ & 79,1758 & 33,5594 & 57,9154 & 59,7018 & 15,1620 & 100 & 35 & 62 & 59 & 20 \\
\hline$(6)$ & 96,0888 & 23,1634 & 60,3026 & 62,0835 & 22,8206 & 99 & 40 & 73 & 80 & 21 \\
\hline$(7)$ & 80,4260 & 17,2251 & 52,8658 & 56,5581 & 22,0383 & 93 & 40 & 69 & 69 & 18 \\
\hline$(8)$ & 68,0831 & 37,5589 & 50,9932 & 47,3031 & 10,7512 & 100 & 46 & 77 & 77 & 18 \\
\hline$(9)$ & 69,6377 & 37,9858 & 53,2974 & 53,6688 & 10,5811 & 100 & $\mathbf{3 3}$ & $\mathbf{5 9}$ & $\mathbf{5 1}$ & 21 \\
\hline média & 75,7096 & 29,4556 & 52,7817 & 52,2786 & 15,3510 & 97 & 41 & 69 & 69 & 19 \\
\hline
\end{tabular}

Tabela 10: Estudo comparativo de resultados de otimização entre PSO, algoritmos genéticos (AGs), programação evolutiva (PE) e estratégia evolutiva (EE) com mecanismos de auto-adaptação.

\begin{tabular}{|c|c|c|c|c|c|}
\hline caso 1(b) & \multicolumn{5}{|c|}{ função objetivo } \\
\hline método de otimização & máximo & mínimo & médio & mediana & desvio padrão \\
\hline PSO(9) - tabela 5 & 0,2430 & 0,0850 & 0,1661 & 0,1710 & 0,0520 \\
\hline AG & 0,4071 & 0,1620 & 0,2293 & 0,2371 & 0,0681 \\
\hline PE & 0,3272 & 0,0591 & 0,1899 & 0,1981 & 0,0701 \\
\hline EE & 0,2617 & 0,0616 & 0,1829 & 0,1905 & 0,0829 \\
\hline caso 2 & \multicolumn{5}{|c|}{ função objetivo } \\
\hline método de otimização & máximo & mínimo & médio & mediana & desvio padrão \\
\hline PSO(4) - tabela 9 & 61,2619 & 15,8254 & 45,8977 & 46,4360 & 12,8982 \\
\hline AG & 92,1131 & 46,3641 & 70,6868 & 69,6558 & 12,5333 \\
\hline PE & 113,1821 & 37,8441 & 73,2459 & 70,6492 & 26,1091 \\
\hline EE & 87,7153 & 31,5241 & 62,9151 & 67,7126 & 15,1654 \\
\hline
\end{tabular}

